\title{
DYNAMICAL ZETA FUNCTIONS, CONGRUENCES IN NIELSEN THEORY AND REIDEMEISTER TORSION
}

\author{
ALEXANDER FEL'SHTYN \\ Fachbereich Mathematik, Universität Greifswald \\ Jahnstraße 15a, D-17487 Greifswald, Germany \\ E-mail: felshtyn@stubber.math-inf.uni-greifswald.de \\ RICHARD HILL \\ Max-Planck-Institut für Mathematik \\ Gottfried-Claren-Strasse 26, D-5300 Bonn 3, Germany \\ E-mail: hill@mpim-bonn.mpg.de
}

\begin{abstract}
In this paper we prove trace formulas for the Reidemeister numbers of group endomorphisms and the rationality of the Reidemeister zeta function in the following cases: the group is finitely generated and the endomorphism is eventually commutative; the group is finite; the group is a direct sum of a finite group and a finitely generated free Abelian group; the group is finitely generated, nilpotent and torsion free. We connect the Reidemeister zeta function of an endomorphism of a direct sum of a finite group and a finitely generated free Abelian group with the Lefschetz zeta function of the unitary dual map, and as a consequence obtain a connection of the Reidemeister zeta function with Reidemeister torsion. We also prove congruences for Reidemeister numbers which are the same as those found by Dold for Lefschetz numbers.
\end{abstract}

1. Introduction. We assume everywhere $X$ to be a connected, compact polyhedron and $f: X \rightarrow X$ to be a continuous map. Taking a dynamical point on view, we consider the iterates of $f$. In the theory of discrete dynamical systems the following zeta functions are known: the Artin-Mazur zeta function

$$
\zeta_{f}(z):=\exp \left(\sum_{n=1}^{\infty} \frac{F\left(f^{n}\right)}{n} z^{n}\right),
$$

1991 Mathematics Subject Classification: Primary 58F20; Secondary 55M20, 57Q10.

Part of this work was conducted during the authors' stay in Sonderforschungsbereich 170 "Geometrie und Analysis", Mathematisches Institut der Georg August Universität zu Göttingen.

The paper is in final form and no version of it will be published elsewhere. 
where $F\left(f^{n}\right)$ is the number of isolated fixed points of $f^{n}$; the Ruelle zeta function [44]

$$
\zeta_{f}^{g}(z):=\exp \left(\sum_{n=1}^{\infty} \frac{z^{n}}{n} \sum_{x \in F i x\left(f^{n}\right)} \prod_{k=0}^{n-1} g\left(f^{k}(x)\right)\right),
$$

where $g: X \rightarrow \mathbb{C}$ is a weight function (if $g=1$ we recover $\zeta_{f}(z)$ ); the Lefschetz zeta function

$$
L_{f}(z):=\exp \left(\sum_{n=1}^{\infty} \frac{L\left(f^{n}\right)}{n} z^{n}\right)
$$

where

$$
L\left(f^{n}\right):=\sum_{k=0}^{\operatorname{dim} X}(-1)^{k} \operatorname{Tr}\left[f_{* k}^{n}: H_{k}(X ; \mathbb{Q}) \rightarrow H_{k}(X ; \mathbb{Q})\right]
$$

are the Lefschetz numbers of the iterates of $f$; reduced mod 2 Artin-Mazur and Lefschetz zeta functions [20]; twisted Artin-Mazur and Lefschetz zeta functions [22], which have coefficients in the group rings $\mathbb{Z} H$ of an Abelian group $H$.

The above zeta functions are directly analogous to the Hasse-Weil zeta function of an algebraic manifold over finite fields [49]. The Lefschetz zeta function is always a rational function of $z$ and is given by the formula:

$$
L_{f}(z)=\prod_{k=0}^{\operatorname{dim} X} \operatorname{det}\left(I-f_{* k} \cdot z\right)^{(-1)^{k+1}} .
$$

This immediately follows from the trace formula for the Lefschetz numbers of the iterates of $f$. The Artin-Mazur zeta function has a positive radius of convergence for a dense set in the space of smooth maps of a compact smooth manifold to itself [2]. Manning proved the rationality of the Artin-Mazur zeta function for diffeomorphisms of a compact smooth manifold satisfying Smale's Axiom A [34].

The Artin-Mazur zeta function and its modification count periodic points of a map geometrically, the Lefschetz type zeta functions do this algebraically (with weight given by index theory). Another way to count the periodic points is given by Nielsen theory.

Let $p: \tilde{X} \rightarrow X$ be the universal covering of $X$ and $\tilde{f}: \tilde{X} \rightarrow \tilde{X}$ a lifting of $f$, i.e. $p \circ \tilde{f}=f \circ p$. Two liftings $\tilde{f}$ and $\tilde{f}^{\prime}$ are called conjugate if there is a $\gamma \in \Gamma \cong \pi_{1}(X)$ such that $\tilde{f}^{\prime}=\gamma \circ \tilde{f} \circ \gamma^{-1}$. The subset $p(\operatorname{Fix}(\tilde{f})) \subset \operatorname{Fix}(f)$ is called the fixed point class of $f$ determined by the lifting class $[\tilde{f}]$. A fixed point class is called essential if its index is nonzero. The number of lifting classes of $f$ (and hence the number of fixed point classes, empty or not) is called the Reidemeister number of $f$, denoted $R(f)$. This is a positive integer or infinity. The number of essential fixed point classes is called the Nielsen number of $f$, denoted by $N(f)$. The Nielsen number is always finite. $R(f)$ and $N(f)$ are homotopy type invariants. In the category of compact, connected polyhedra the Nielsen number of a map is equal to the least number of fixed points of maps with the same homotopy type as $f$. Let $G$ be a group and $\phi: G \rightarrow G$ an endomorphism. Two elements $\alpha, \alpha^{\prime} \in G$ are said to be $\phi$-conjugate iff there exists $\gamma \in G$ such that $\alpha^{\prime}=\gamma \cdot \alpha \cdot \phi(\gamma)^{-1}$. The number of $\phi$-conjugacy classes is called the Reidemeister number of $\phi$, denoted by $R(\phi)$. We shall also write $\mathcal{R}(\phi)$ for the set of $\phi$-conjugacy classes of elements of $G$. 
If we consider the iterates of $f$ and $\phi$, we may define several zeta functions connected with Nielsen fixed point theory (see $[11,12,14,15,19])$. We assume throughout this article that $R\left(f^{n}\right)<\infty$ and $R\left(\phi^{n}\right)<\infty$ for all $n>0$. The Reidemeister zeta functions of $f$ and $\phi$ and the Nielsen zeta function of $f$ are defined as power series:

$$
\begin{gathered}
R_{f}(z):=\exp \left(\sum_{n=1}^{\infty} \frac{R\left(f^{n}\right)}{n} z^{n}\right), \quad R_{\phi}(z):=\exp \left(\sum_{n=1}^{\infty} \frac{R\left(\phi^{n}\right)}{n} z^{n}\right) \\
N_{f}(z):=\exp \left(\sum_{n=1}^{\infty} \frac{N\left(f^{n}\right)}{n} z^{n}\right) .
\end{gathered}
$$

$R_{f}(z)$ and $N_{f}(z)$ are homotopy invariants. The Nielsen zeta function $N_{f}(z)$ has a positive radius of convergence which has a sharp estimate in terms of the topological entropy of the map $f$ [19]. In section 6 we propose another proof of positivity of the radius and give an exact algebraic lower estimation for the radius using the Reidemeister trace formula for the generalized Lefschetz numbers. In section 9 we prove the same result for the radius of convergence of the minimal dynamical zeta function.

An endomorphism $\phi: G \rightarrow G$ is said to be eventually commutative if there exists a natural number $n$ such that the subgroup $\phi^{n}(G)$ is commutative. A map $f: X \rightarrow X$ is said to be eventually commutative if the induced endomorphism on the fundamental group is eventually commutative. We begin the article by proving in section 1 the trace formula for the Reidemeister numbers in the following cases: $G$ is finitely generated and $\phi$ is eventually commutative; $G$ is finite; $G$ is a direct sum of a finite group and a finitely generated free Abelian group; $G$ is a finitely generated torsion free nilpotent group.

This result was previously known only for the finitely generated free Abelian group [15]. As a consequence, we obtain in section 2, under the same conditions on the fundamental group of $X$, the trace formula for the Reidemeister numbers of a continuous map and in section 3 under suitable conditions the trace formulas for the Nielsen numbers of a continuous map.

The trace formula for the Reidemeister numbers implies the rationality of the Reidemeister zeta function. In section 5 we prove the rationality and functional equation for the Reidemeister zeta function of an endomorphism of a finitely generated torsion free nilpotent group and of a direct sum of a finite group and a finitely generated free Abelian group. We give also a new proof of the rationality of $R_{\phi}(z)$ in the case when $G$ is finitely generated and $\phi$ is eventually commutative and in the case when $G$ is finite.

In section 5.5 we describe some conjectures on how the Reidemeister zeta functions should look in general, largely in terms of the growth of the group. In particular we expect that for polynomial growth groups some power of the Reidemeister zeta function is a rational function.

In section 6 we continue to study analytical properties of the Nielsen zeta function. We would like to mention that in all known cases the Nielsen zeta function is a nice function. By this we mean that it is a product of an exponential of a polynomial with a function some power of which is rational. Maybe this is a general pattern.

In his article [7], Dold found a remarkable arithmetical property of the Lefschetz numbers for the iterations of a map $f$. He proved the following formula 


$$
\sum_{d \mid n} \mu(d) \cdot L\left(f^{n / d}\right) \equiv 0 \bmod n
$$

where $n$ is any natural number and $\mu$ is the Möbius function. In the case that $n$ is prime, this result was obtained by Zabreǐko, Krasnosel'skiı̌ [50] and Steinlein [46]. In section 7 we prove, under additional conditions, similar formulae:

$$
\sum_{d \mid n} \mu(d) \cdot R\left(\phi^{n / d}\right) \equiv 0 \bmod n, \quad \sum_{d \mid n} \mu(d) \cdot R\left(f^{n / d}\right) \equiv 0 \bmod n
$$

for the Reidemeister numbers of the iterations of a group endomorphism $\phi$ and a map $f$. This result implies, in the special cases, the corresponding congruences for the Nielsen numbers.

In section 8 we explain how dynamical zeta functions give rise to the Reidemeister torsion, a very important topological invariant. In 1935 Reidemeister [41] classified up to $P L$ equivalence the lens spaces $S^{3} / \Gamma$ where $\Gamma$ is a finite cyclic group of fixed point free orthogonal transformations. He used a certain new invariant which was quickly extended by Franz [21], who used it to classify the generalized lens spaces $S^{2 n+1} / \Gamma$. This invariant is a ratio of determinants concocted from a $\Gamma$-equivariant chain complex of $S^{2 n+1}$ and a nontrivial character $\rho: \Gamma \rightarrow U(1)$ of $\Gamma$. Such a $\rho$ determines a flat bundle $E$ over $S^{2 n+1} / \Gamma$ such that $E$ has holonomy $\rho$. The new invariant is now called the Reidemeister torsion or $R$-torsion of $E$. The Reidemeister torsion is closely related to the $K_{1}$ groups of algebraic $K$-theory. The results of Reidemeister and Franz were extended by de Rham [42] to spaces of constant curvature +1 .

Later Milnor [36] identified the Reidemeister torsion with the Alexander polynomial, which plays a fundamental role in the theory of knots and links. After that Cheeger [6] and Müller [37] proved that the Reidemeister torsion coincides with the analytical torsion of Ray and Singer [40].

Recently a connection between the Lefschetz type dynamical zeta functions and the Reidemeister torsion was established by D. Fried [23, 24]. The work of Milnor [35] was the first indication that such a connection exists.

In section 8 we establish a connection between the Reidemeister torsion and Reidemeister zeta function. We obtain an expression for the Reidemeister torsion of the mapping torus of the dual map of an endomorphism of a direct sum of a finite group and a finitely generated free Abelian group, in terms of the Reidemeister zeta function of the endomorphism. The result is obtained by expressing the Reidemeister zeta function in terms of the Lefschetz zeta function of the dual map, and then applying the theorem of D. Fried. This means that the Reidemeister torsion counts the fixed point classes of all iterates of map $f$, i.e. periodic point classes of $f$. This was previously known for the finitely generated Abelian groups and finite groups [17].

We would like to thank J. Eichhorn, D. Fried, M. L. Gromov and B. B. Venkov for valuable conversations and comments. Alexander Fel'shtyn would like to thank Institut des Hautes Etudes Scientifiques for their kind hospitality and support. Richard Hill would like to thank the Max Planck Institut für Mathematik in Bonn for their hospitality. 


\section{Trace formula for the Reidemeister numbers of a group endomorphism}

2.1. Pontryagin duality. Let $G$ be a locally compact Abelian topological group. We write $\hat{G}$ for the set of continuous homomorphisms from $G$ to the circle $U(1)=\{z \in \mathbb{C}$ : $|z|=1\}$. This is a group with pointwise multiplication. We call $\hat{G}$ the Pontryagin dual of $G$. When we equip $\hat{G}$ with the compact-open topology it becomes a locally compact Abelian topological group. The dual of the dual of $G$ is canonically isomorphic to $G$.

A continuous endomorphism $f: G \rightarrow G$ gives rise to a continuous endomorphism $\hat{f}: \hat{G} \rightarrow \hat{G}$ defined by

$$
\hat{f}(\chi):=\chi \circ f .
$$

There is a 1-1 correspondence between the closed subgroups $H$ of $G$ and the quotient groups $\hat{G} / H^{*}$ of $\hat{G}$ for which $H^{*}$ is closed in $\hat{G}$. This correspondence is given by the following:

$$
H \leftrightarrow \hat{G} / H^{*}, \quad H^{*}:=\{\chi \in \hat{G} \mid H \subset \operatorname{ker} \chi\} .
$$

Under this correspondence, $\hat{G} / H^{*}$ is canonically isomorphic to the Pontryagin dual of $H$. If we identify $G$ canonically with the dual of $\hat{G}$ then we have $H^{* *}=H$. If $G$ is a finitely generated Abelian group then a homomorphism $\chi: G \rightarrow U(1)$ is completely determined by its values on a basis of $G$, and these values may be chosen arbitrarily. The dual of $G$ is thus a torus whose dimension is equal to the rank of $G$.

If $G=\mathbb{Z} / n \mathbb{Z}$ then the elements of $\hat{G}$ are of the form $x \rightarrow e^{2 \pi i y x / n}$ with $y \in$ $\{1,2, \ldots, n\}$. A cyclic group is therefore (non-canonically) isomorphic to itself.

The dual of $G_{1} \oplus G_{2}$ is canonically isomorphic to $\hat{G}_{1} \oplus \hat{G}_{2}$. From this we see that any finite Abelian group is (non-canonically) isomorphic to its own Pontryagin dual group, and that the dual of any finitely generated discrete Abelian group is the direct sum of a torus and a finite group.

Proofs of all these statements may be found for example in [43]. We shall need the following statement:

Proposition 1. Let $\phi: G \rightarrow G$ be an endomorphism of an Abelian group $G$. Then the kernel $\operatorname{ker}[\hat{\phi}: \hat{G} \rightarrow \hat{G}]$ is canonically isomorphic to the Pontryagin dual of Coker $\phi$.

ProOF. We construct the isomorphism explicitly. Let $\chi$ be in the dual of $\operatorname{Coker}(\phi$ : $G \rightarrow G)$. In that case $\chi$ is a homomorphism $\chi: G / \operatorname{Im}(\phi) \rightarrow U(1)$. There is therefore an induced map $\bar{\chi}: G \rightarrow U(1)$ which is trivial on $\operatorname{Im}(\phi)$. This means that $\bar{\chi} \circ \phi$ is trivial, or in other words $\hat{\phi}(\bar{\chi})$ is the identity element of $\hat{G}$. We therefore have $\bar{\chi} \in \operatorname{ker}(\hat{\phi})$.

If on the other hand we begin with $\bar{\chi} \in \operatorname{ker}(\hat{\phi})$, then it follows that $\chi$ is trivial on $\operatorname{Im} \phi$, and so $\bar{\chi}$ induces a homomorphism $\chi: G / \operatorname{Im}(\phi) \rightarrow U(1)$ and $\chi$ is then in the dual of Coker $\phi$. The correspondence $\chi \leftrightarrow \bar{\chi}$ is clearly a bijection.

2.2. Irreducible representations and the unitary dual of $G$. Let $V$ be a Hilbert space. A unitary representation of $G$ on $V$ is a homomorphism $\rho: G \rightarrow \mathrm{U}(V)$ where $\mathrm{U}(V)$ is the group of unitary transformations of $V$. Two of these $\rho_{1}: G \rightarrow \mathrm{U}\left(V_{1}\right)$ and $\rho_{2}: G \rightarrow \mathrm{U}\left(V_{2}\right)$ are said to be equivalent if there is a Hilbert space isomorphism $V_{1} \cong V_{2}$ which commutes with the $G$-actions. A representation $\rho: G \rightarrow \mathrm{U}(V)$ is said to be irreducible if there is no decomposition $V \cong V_{1} \oplus V_{2}$ in which $V_{1}$ and $V_{2}$ are non-zero, closed $G$-submodules of $V$. 
One defines the unitary dual $\hat{G}$ of $G$ to be the set of all equivalence classes of irreducible, unitary representations of $G$.

If $\rho: G \rightarrow \mathrm{U}(V)$ is a representation then $\rho \circ \phi: G \rightarrow \mathrm{U}(V)$ is also a representation, which we shall denote $\hat{\phi}(\rho)$. If $\rho_{1}$ and $\rho_{2}$ are equivalent then $\hat{\phi}\left(\rho_{1}\right)$ and $\hat{\phi}\left(\rho_{2}\right)$ are equivalent. Therefore the endomorphism $\phi$ induces a map $\hat{\phi}: \hat{G} \rightarrow \hat{G}$ from the unitary dual to itself.

Definition 1. Define the number \# Fix $(\hat{\phi})$ to be the number of fixed points of the induced map $\hat{\phi}: \hat{G} \rightarrow \hat{G}$. We shall write $\mathcal{S}(\phi)$ for the set of fixed points of $\hat{\phi}$. Thus $\mathcal{S}(\phi)$ is the set of equivalence classes of irreducible representations $\rho: G \rightarrow \mathrm{U}(V)$ such that there is a transformation $M \in \mathrm{U}(V)$ satisfying

$$
\forall x \in G, \quad \rho(\phi(x))=M \cdot \rho(x) \cdot M^{-1} .
$$

Note that if $\phi$ is an inner automorphism $x \mapsto g x g^{-1}$ then we have for any representation $\rho$,

$$
\rho(\phi(x))=\rho(g) \cdot \rho(x) \cdot \rho(g)^{-1},
$$

implying that the class of $\rho$ is fixed by the induced map. Thus for an inner automorphism the induced map is trivial and \# $\operatorname{Fix}(\hat{\phi})$ is the cardinality of $\hat{G}$. When $G$ is Abelian the group $\hat{G}$ is the Pontryagin dual of $G$.

2.3. Eventually commutative endomorphisms. An endomorphism $\phi: G \rightarrow G$ is said to be eventually commutative if there exists a natural number $n$ such that the subgroup $\phi^{n}(G)$ is commutative. If $\phi$ is an endomorphism of an Abelian group $G$ then $x$ and $y$ are $\phi$-conjugate iff $x-y=\phi(g)-g$ for some $g \in G$. Therefore $R(\phi)$ is the number of cosets of the image of the endomorphism $(\phi-1): G \rightarrow G$. Then

$$
R(\phi)=\# \operatorname{Coker}(1-\phi)
$$

We are now ready to compare the Reidemeister number of an endomorphism $\phi$ with the Reidemeister number of $H_{1}(\phi): H_{1}(G) \rightarrow H_{1}(G)$, where $H_{1}=H_{1}^{G p}$ is the first integral homology functor from groups to Abelian groups.

LEMMA 1 ([28]). If $\phi: G \rightarrow G$ is eventually commutative, then

$$
R(\phi)=R\left(H_{1}(\phi)=\# \operatorname{Coker}\left(1-H_{1}(\phi)\right) .\right.
$$

This means that to find out about the Reidemeister numbers of eventually commutative endomorphisms, it is sufficient to study the Reidemeister numbers of endomorphisms of Abelian groups. For the rest of this section $G$ will be a finitely generated Abelian group.

Lemma $2([15])$. Let $\phi: \mathbb{Z}^{k} \rightarrow \mathbb{Z}^{k}$ be a group endomorphism. Then

$$
R(\phi)=(-1)^{r+p} \sum_{i=0}^{k}(-1)^{i} \operatorname{Tr}\left(\Lambda^{i} \phi\right)
$$

where $p$ the number of $\mu \in \operatorname{Spec} \phi$ such that $\mu<-1$, and $r$ the number of real eigenvalues of $\phi$ whose absolute value is $>1 . \Lambda^{i}$ denotes the exterior power.

Now let $\phi$ be an endomorphism of a finite Abelian group $G$. Let $V$ be the complex vector space of complex valued functions on the group $G$. The map $\phi$ induces a linear 
map $A: V \rightarrow V$ defined by

$$
A(f):=f \circ \phi
$$

Lemma 3. Let $\phi: G \rightarrow G$ be an endomorphism of a finite Abelian group $G$. Then

$$
R(\phi)=\operatorname{Tr} A .
$$

We give two proofs of this lemma in this article. The first proof is given here and the second proof is a special case of the proof of theorem 4 .

Proof. The characteristic functions of the elements of $G$ form a basis of $V$, and are mapped to one another by $A$ (the map need not be a bijection). Therefore the trace of $A$ is the number of elements of this basis which are fixed by $A$. On the other hand, since $G$ is Abelian, we have

$$
\begin{aligned}
R(\phi) & =\# \operatorname{Coker}(1-\phi)=\# G / \# \operatorname{Im}(1-\phi)=\# G / \#(G / \operatorname{ker}(1-\phi)) \\
& =\# G /(\# G / \# \operatorname{ker}(1-\phi))=\# \operatorname{ker}(1-\phi)=\# \operatorname{Fix}(\phi) .
\end{aligned}
$$

We therefore have $R(\phi)=\# \operatorname{Fix}(\phi)=\operatorname{Tr} A$.

For a finitely generated Abelian group $G$ we define the finite subgroup $G^{\text {finite }}$ to be the subgroup of torsion elements of $G$. We denote the quotient $G^{\infty}:=G / G^{\text {finite }}$. The group $G^{\infty}$ is torsion free. Since the image of any torsion element by a homomorphism must be a torsion element, the endomorphism $\phi: G \rightarrow G$ induces endomorphisms

$$
\phi^{\text {finite }}: G^{\text {finite }} \longrightarrow G^{\text {finite }}, \quad \phi^{\infty}: G^{\infty} \longrightarrow G^{\infty} .
$$

As above, the map $\phi^{\text {finite }}$ induces a linear map $A: V \rightarrow V$, where $V$ is the complex vector space of complex valued functions on the group $G^{\text {finite }}$.

THEOREM 1. If $G$ is a finitely generated Abelian group and $\phi$ an endomorphism of $G$, then

$$
R(\phi)=(-1)^{r+p} \sum_{i=0}^{k}(-1)^{i} \operatorname{Tr}\left(\Lambda^{i} \phi^{\infty} \otimes A\right)
$$

where $k$ is $\operatorname{rg} G^{\infty}$, $p$ the number of $\mu \in \operatorname{Spec} \phi^{\infty}$ such that $\mu<-1$, and $r$ the number of real eigenvalues of $\phi^{\infty}$ whose absolute value is $>1$.

Proof. By proposition 1, the cokernel of $(1-\phi): G \rightarrow G$ is the Pontryagin dual of the kernel of the dual map $(\widehat{1-\phi}): \hat{G} \rightarrow \hat{G}$. Since Coker $(1-\phi)$ is finite, we have

$$
\# \operatorname{Coker}(1-\phi)=\# \operatorname{ker}(\widehat{1-\phi})
$$

The map $\widehat{1-\phi}$ is equal to $\hat{1}-\hat{\phi}$. Its kernel is thus the set of fixed points of the map $\hat{\phi}: \hat{G} \rightarrow \hat{G}$. We therefore have

$$
R(\phi)=\# \operatorname{Fix}(\hat{\phi}: \hat{G} \rightarrow \hat{G}) .
$$

The dual group of $G^{\infty}$ is a torus whose dimension is the rank of $G$. This is canonically a closed subgroup of $\hat{G}$. We shall denote it $\hat{G}_{0}$. The quotient $\hat{G} / \hat{G}_{0}$ is canonically isomorphic to the dual of $G^{\text {finite }}$. It is therefore finite. From this we know that $\hat{G}$ is a union of finitely many disjoint tori. We shall call these tori $\hat{G}_{0}, \ldots, \hat{G}_{t}$. 
We shall call a torus $\hat{G}_{i}$ periodic if there is an iteration $\hat{\phi}^{s}$ such that $\hat{\phi}^{s}\left(\hat{G}_{i}\right) \subset \hat{G}_{i}$. If this is the case, then the map $\hat{\phi}^{s}: \hat{G}_{i} \rightarrow \hat{G}_{i}$ is a translation of the map $\hat{\phi}^{s}: \hat{G}_{0} \rightarrow \hat{G}_{0}$ and has the same number of fixed points as this map. If $\hat{\phi}^{s}\left(\hat{G}_{i}\right) \not \subset \hat{G}_{i}$ then $\hat{\phi}^{s}$ has no fixed points in $\hat{G}_{i}$. From this we see

$$
\# \operatorname{Fix}(\hat{\phi}: \hat{G} \rightarrow \hat{G})=\# \operatorname{Fix}\left(\hat{\phi}: \hat{G}_{0} \rightarrow \hat{G}_{0}\right) \times \#\left\{\hat{G}_{i} \mid \hat{\phi}\left(\hat{G}_{i}\right) \subset \hat{G}_{i}\right\} .
$$

We now rephrase this

$$
\# \operatorname{Fix}(\hat{\phi}: \hat{G} \rightarrow \hat{G})=\# \operatorname{Fix}\left(\widehat{\phi^{\infty}}: \hat{G}_{0} \rightarrow \hat{G}_{0}\right) \times \# \operatorname{Fix}\left(\phi^{\text {finite }}: \hat{G} /\left(\hat{G}_{0}\right) \rightarrow \hat{G} /\left(\hat{G}_{0}\right)\right)
$$

From this we have the product formula for Reidemeister numbers

$$
R(\phi)=R\left(\phi^{\infty}\right) \cdot R\left(\phi^{\text {finite }}\right) .
$$

The trace formula for $R(\phi)$ follows from the previous two lemmas and the formula

$$
\operatorname{Tr}\left(\Lambda^{i} \phi^{\infty}\right) \cdot \operatorname{Tr}(A)=\operatorname{Tr}\left(\Lambda^{i} \phi^{\infty} \otimes A\right) .
$$

In the paper [17] we have connected the Reidemeister number of an endomorphism $\phi$ with the Lefschetz number of the dual map. From this we have the following trace formula:

THEOREM $2([17])$. Let $\phi: G \rightarrow G$ be an endomorphism of a finitely generated Abelian group. Then

$$
R(\phi)=|L(\hat{\phi})|=(-1)^{r+p} \sum_{k=0}^{\operatorname{dim} \hat{G}}(-1)^{k} \operatorname{Tr}\left[\hat{\phi}_{* k}: H_{k}(\hat{G} ; \mathbb{Q}) \rightarrow H_{k}(\hat{G} ; \mathbb{Q})\right]
$$

where $\hat{\phi}$ is the continuous endomorphism of $\hat{G}$ defined in $\$ 2.2$ and $L(\hat{\phi})$ is the Lefschetz number of $\hat{\phi}$ thought of as a self-map of the topological space $\hat{G}$ and $r$ and $p$ are the constants described in theorem 1. If $G$ is finite then this reduces to

$$
R(\phi)=L(\hat{\phi})=\operatorname{Tr}\left[\hat{\phi}_{* 0}: H_{0}(\hat{G} ; \mathbb{Q}) \rightarrow H_{0}(\hat{G} ; \mathbb{Q})\right] .
$$

2.4. Endomorphisms of finite groups. In this section we consider finite non-Abelian groups. We shall write the group law multiplicatively. We generalize our results on endomorphisms of finite Abelian groups to endomorphisms of finite non-Abelian groups. We shall write $\{g\}$ for the $\phi$-conjugacy class of an element $g \in G$. We shall write $\langle g\rangle$ for the ordinary conjugacy class of $g$ in $G$. We first note that if $\phi$ is an endomorphism of a group $G$ then $\phi$ maps conjugate elements to conjugate elements. It therefore induces an endomorphism of the set of conjugacy classes of elements of $G$. If $G$ is Abelian then a conjugacy class of element consists of a single element. The following is thus an extension of lemma 3 :

Theorem 3 ([16]). Let $G$ be a finite group and let $\phi: G \rightarrow G$ be an endomorphism. Then $R(\phi)$ is the number of ordinary conjugacy classes $\langle x\rangle$ in $G$ such that

$$
\langle\phi(x)\rangle=\langle x\rangle .
$$

So, we have proved that the number of congruence classes in $G$ which are mapped to themselves by $\phi$ is precisely the Reidemeister number $R(\phi)$. 
Corollary 1. Suppose that $\phi_{1}$ and $\phi_{2}$ are two endomorphisms of a finite group $G$ with

$$
\forall g \in G, \phi_{1}(g)=h \phi_{2}(g) h^{-1}
$$

for some fixed element $h \in G$. Then $R\left(\phi_{1}\right)=R\left(\phi_{2}\right)$.

COROLlarY 2. Let $\phi$ be an inner automorphism. Then $R(\phi)=b$ where $b$ is the number of conjugacy classes in the group. In particular, all but finitely many of the symmetric and alternating groups have the property that any automorphism is an inner automorphism, and so this corollary applies.

REMARK 1. If we think of the set of conjugacy classes in $G$ as a discrete set then the Reidemeister number of $\phi$ is equal to the Lefschetz number of the induced map on the conjugacy classes.

Let $W$ be the complex vector space of complex valued class functions on the group $G$. A class function is a function which takes the same value on every element of a usual congruence class. The map $\phi$ induces a linear map $B: W \rightarrow W$ defined by

$$
B(f):=f \circ \phi .
$$

Theorem 4. Let $\phi: G \rightarrow G$ be an endomorphism of a finite group $G$. Then

$$
R(\phi)=\# \operatorname{Fix}(\hat{\phi})=\operatorname{Tr} B .
$$

Proof. We shall calculate the trace of $B$ in two ways. The characteristic functions of the congruence classes in $G$ form a basis of $W$, and are mapped to one another by $B$ (the map need not be a bijection). Therefore the trace of $B$ is the number of elements of this basis which are fixed by $B$. By theorem 3, this is equal to the Reidemeister number of $\phi$. Another basis of $W$, which is also mapped to itself by $B$, is the set of traces of irreducible representations of $G$ (see [31] chapter XVIII). From this it follows that the trace of $B$ is the number of irreducible representations $\rho$ of $G$ such that $\rho$ has the same trace as $\hat{\phi}(\rho)$. However, representations of finite groups are characterized up to equivalence by their traces. Therefore the trace of $B$ is equal to the number of fixed points of $\hat{\phi}$.

REMARK 2. By specialising to the case when $G$ is finite and $\phi$ is the identity map, we obtain the classical Burnside theorem equating the number of irreducible representations of a finite group with the number of conjugacy classes of the group.

2.5. Endomorphisms of the direct sum of a free Abelian and a finite group. In this section let $F$ be a finite group and $r$ a natural number. The group $G$ will be

$$
G=\mathbb{Z}^{r} \oplus F \text {. }
$$

We shall describe the Reidemeister numbers of an endomorphism $\phi: G \rightarrow G$. The torsion elements of $G$ are exactly the elements of the finite, normal subgroup $F$. For this reason we have $\phi(F) \subset F$. Let $\phi^{\text {finite }}: F \rightarrow F$ be the restriction of $\phi$ to $F$, and let $\phi^{\infty}: G / F \rightarrow G / F$ be the induced map on the quotient group.

Let $\operatorname{pr}_{\mathbb{Z}^{r}}: G \rightarrow \mathbb{Z}^{r}$ and $\operatorname{pr}_{F}: G \rightarrow F$ be the projections onto $\mathbb{Z}^{r}$ and $F$. Then the composition

$$
\operatorname{pr}_{\mathbb{Z}^{r}} \circ \phi: \mathbb{Z}^{r} \rightarrow G \rightarrow \mathbb{Z}^{r}
$$


is an endomorphism of $\mathbb{Z}^{r}$, which is given by some matrix $M \in M_{r}(\mathbb{Z})$. We denote by $\psi: \mathbb{Z}^{r} \rightarrow F$ the other component of the restriction of $\phi$ to $\mathbb{Z}^{r}$, i.e.

$$
\psi(v)=\operatorname{pr}_{F}(\phi(v)) .
$$

We therefore have for any element $(v, f) \in G$

$$
\phi(v, f)=(M \cdot v, \psi(v) \phi(f)) .
$$

Lemma 4. Let $g_{1}=\left(v_{1}, f_{1}\right)$ and $g_{2}=\left(v_{2}, f_{2}\right)$ be two elements of $G$. Then $g_{1}$ and $g_{2}$ are $\phi$-conjugate iff

$$
v_{1} \equiv v_{2} \bmod (1-M) \mathbb{Z}^{r}
$$

and there is an $h \in F$ with

$$
h f_{1}=f_{2} \phi\left((1-M)^{-1}\left(v_{2}-v_{1}\right)\right) \phi(h) .
$$

Proof. Suppose $g_{1}$ and $g_{2}$ are $\phi$-conjugate. Then there is a $g_{3}=(w, h) \in G$ with $g_{3} g_{1}=g_{2} \phi\left(g_{3}\right)$. Therefore

$$
\left(w+v_{1}, h f_{1}\right)=\left(v_{2}+M \cdot w, f_{2} \psi(w) \phi(h)\right) .
$$

Comparing the first components we obtain $(1-M) \cdot w=v_{2}-v_{1}$ from which it follows that $v_{1}$ is congruent to $v_{2}$ modulo $(1-M) \mathbb{Z}^{r}$. Substituting $(1-M)^{-1}\left(v_{2}-v_{1}\right)$ for $w$ in the second component we obtain the second relation in the lemma. The argument can easily be reversed to give the converse.

Proposition 2. In the notation described above

$$
R(\phi)=R\left(\phi^{\text {finite }}\right) \times R\left(\phi^{\infty}\right) .
$$

Proof. We partition the set $\mathcal{R}(\phi)$ of $\phi$-conjugacy classes of elements of $G$ into smaller sets:

$$
\mathcal{R}(\phi)=\bigcup_{v \in \mathbb{Z}^{r} /(1-M) \mathbb{Z}^{r}} \mathcal{R}(v)
$$

where $\mathcal{R}(v)$ is the set of $\phi$-conjugacy classes $\{(w, f)\}_{\phi}$ for which $w$ is congruent to $v$ modulo $(1-M) \mathbb{Z}^{r}$. It follows from the previous lemma that this is a partition. Now suppose $\{(w, f)\}_{\phi} \in \mathcal{R}(v)$. We will show that $\{(w, f)\}_{\phi}=\left\{\left(v, f^{*}\right)\right\}_{\phi}$ for some $f^{*} \in F$. This follows by setting $f^{*}=f \psi\left((1-M)^{-1}(w-v)\right)$ and applying the previous lemma with $h=i d$. Therefore $\mathcal{R}(v)$ is the set of $\phi$-conjugacy classes $\{(v, f)\}_{\phi}$ with $f \in F$. From the previous lemma it follows that $\left(v, f_{1}\right)$ and $\left(v, f_{2}\right)$ are $\phi$-conjugate iff there is an $h \in F$ with

$$
h f_{1}=f_{2} \psi(0) \phi(h)=f_{2} \phi(h) .
$$

This just means that $f_{1}$ and $f_{2}$ are $\phi^{\text {finite }}$-conjugate as elements of $F$. From this it follows that $\mathcal{R}(v)$ has cardinality $R\left(\phi^{\text {finite }}\right)$. Since this is independent of $v$, we have

$$
R(\phi)=\sum_{v} R\left(\phi^{\text {finite }}\right)=|\operatorname{det}(1-M)| \times R\left(\phi^{\text {finite }}\right) .
$$

Now consider the map $\phi^{\infty}: G / F \rightarrow G / F$. We have

$$
\phi^{\infty}((v, F))=(M \cdot v, \psi(v) F)=(M \cdot v, F) .
$$


From this it follows that $\phi^{\infty}$ is isomorphic to map $M: \mathbb{Z}^{r} \rightarrow \mathbb{Z}^{r}$. This implies

$$
R\left(\phi^{\infty}\right)=R\left(M: \mathbb{Z}^{r} \rightarrow \mathbb{Z}^{r}\right)
$$

but it is known [16] that $R\left(M: \mathbb{Z}^{r} \rightarrow \mathbb{Z}^{r}\right)=|\operatorname{det}(1-M)|$. Therefore $R(\phi)=R\left(\phi^{\text {finite }}\right) \times$ $R\left(\phi^{\infty}\right)$, proving proposition 3 .

Let $W$ be the complex vector space of complex valued class functions on the group $F$. The map $\phi$ induces a linear map $B: W \rightarrow W$ defined as above in theorem 4 .

TheOREm 5. If $G$ is the direct sum of a free Abelian and a finite group and $\phi$ an endomorphism of $G$, then

$$
R(\phi)=(-1)^{r+p} \sum_{i=0}^{k}(-1)^{i} \operatorname{Tr}\left(\Lambda^{i} \phi^{\infty} \otimes B\right) .
$$

where $k$ is $\operatorname{rg}(G / F), p$ the number of $\mu \in \operatorname{Spec} \phi^{\infty}$ such that $\mu<-1$, and $r$ the number of real eigenvalues of $\phi^{\infty}$ whose absolute value is $>1$.

Proof. This follows from lemma 2 and theorem 4, proposition 2 and the formula

$$
\operatorname{Tr}\left(\Lambda^{i} \phi^{\infty}\right) \cdot \operatorname{Tr}(B)=\operatorname{Tr}\left(\Lambda^{i} \phi^{\infty} \otimes B\right) .
$$

2.6. Endomorphisms of nilpotent groups. In this section we consider finitely generated torsion free nilpotent groups $\Gamma$. It is well known [33] that such a group $\Gamma$ is a uniform discrete subgroup of a simply connected nilpotent Lie group $G$ (uniform means that the coset space $G / \Gamma$ is compact). The coset space $M=G / \Gamma$ is called a nilmanifold. Since $\Gamma=\pi_{1}(M)$ and $M$ is a $K(\Gamma, 1)$, every endomorphism $\phi: \Gamma \rightarrow \Gamma$ can be realized by a self-map $f: M \rightarrow M$ such that $f_{*}=\phi$ and thus $R(f)=R(\phi)$. Any endomorphism $\phi: \Gamma \rightarrow \Gamma$ can be uniquely extended to an endomorphism $F: G \rightarrow G$. Let $\tilde{F}: \tilde{G} \rightarrow \tilde{G}$ be the corresponding Lie algebra endomorphism induced from $F$.

THEOREM 6. If $\Gamma$ is a finitely generated torsion free nilpotent group and $\phi$ an endomorphism of $\Gamma$, then

$$
R(\phi)=(-1)^{r+p} \sum_{i=0}^{m}(-1)^{i} \operatorname{Tr} \Lambda^{i} \tilde{F},
$$

where $m$ is $\operatorname{rg} \Gamma=\operatorname{dim} M, p$ the number of $\mu \in \operatorname{Spec} \tilde{F}$ such that $\mu<-1$, and $r$ the number of real eigenvalues of $\tilde{F}$ whose absolute value is $>1$.

Proof. Let $f: M \rightarrow M$ be a map realizing $\phi$ on a compact nilmanifold $M$ of dimension $m$. We suppose in this article that the Reidemeister number $R(f)=R(\phi)$ is finite. The finiteness of $R(f)$ implies the nonvanishing of the Lefschetz number $L(f)$ [18]. A strengthened version of Anosov's theorem [1] is proven in [38] which states, in particular, that if $L(f) \neq 0$ then $N(f)=|L(f)|=R(f)$. It is well known that $L(f)=$ $\operatorname{det}(\tilde{F}-1)[1]$. From this we have

$$
R(\phi)=R(f)=|L(f)|=|\operatorname{det}(1-\tilde{F})|=(-1)^{r+p} \operatorname{det}(1-\tilde{F})=(-1)^{r+p} \sum_{i=0}^{m}(-1)^{i} \operatorname{Tr} \Lambda^{i} \tilde{F} .
$$


2.7. The trace formulas and group extensions. Suppose we are given a commutative diagram

$$
\begin{aligned}
& G \stackrel{\phi}{\longrightarrow} G \\
& \downarrow p \\
& \bar{G} \stackrel{\bar{\phi}}{\longrightarrow} \bar{G}
\end{aligned}
$$

of groups and homomorphisms. In addition let the sequence

$$
0 \rightarrow H \rightarrow G \stackrel{p}{\rightarrow} \bar{G} \rightarrow 0
$$

be exact. Then $\phi$ restricts to an endomorphism $\left.\phi\right|_{H}: H \rightarrow H$.

Definition 2. The short exact sequence (11) of groups is said to have a normal splitting if there is a section $\sigma: \bar{G} \rightarrow G$ of $p$ such that $\operatorname{Im} \sigma=\sigma(\bar{G})$ is a normal subgroup of $G$. An endomorphism $\phi: G \rightarrow G$ is said to preserve this normal splitting if $\phi$ induces a morphism of (11) with $\phi(\sigma(\bar{G})) \subset \sigma(\bar{G})$.

In this section we study the relation between the trace formulas of type (8) for Reidemeister numbers $R(\phi), R(\bar{\phi})$ and $R\left(\left.\phi\right|_{H}\right)$.

THEOREM 7. Let the sequence (11) have a normal splitting which is preserved by $\phi: G \rightarrow G$. If we have trace formulas of the type (8) for $R(\bar{\phi})$ and $R\left(\left.\phi\right|_{H}\right)$ then we have a trace formula of the same type for $R(\phi)$.

Proof. From the assumptions of the theorem it follows that $R(\phi)=R(\bar{\phi}) \cdot R\left(\left.\phi\right|_{H}\right.$ ) (see [27]). The trace formula for $R(\phi)$ now follows from the linear algebra formula $\operatorname{Tr} A \cdot \operatorname{Tr} B=$ $\operatorname{Tr} A \otimes B$.

Direct sums. If $G=G_{1} \oplus G_{2}$ is a direct sum and if $\phi\left(G_{i}\right) \subset G_{i}$ for $i=1,2$ then it has been shown (see [27]) that $R(\phi)=R\left(\phi_{1}\right) \cdot R\left(\phi_{2}\right)$ where $\phi_{i}$ is the restriction of $\phi$ to $G_{i}$. So if we have a trace formula of type (8) for $R\left(\phi_{1}\right)$ and $R\left(\phi_{2}\right)$ then we have a trace formula for $R(\phi)$.

3. Trace formulas for Reidemeisters numbers of a continuous map. Let $f$ : $X \rightarrow X$ be given, and let a specific lifting $\tilde{f}: \tilde{X} \rightarrow \tilde{X}$ be chosen as reference. Let $\Gamma$ be the group of covering translations of $\tilde{X}$ over $X$. Then every lifting of $f$ can be written uniquely as $\gamma \circ \tilde{f}$, with $\gamma \in \Gamma$. So elements of $\Gamma$ serve as coordinates of liftings with respect to the reference $\tilde{f}$. Now for every $\gamma \in \Gamma$ the composition $\tilde{f} \circ \gamma$ is a lifting of $f$ so there is a unique $\gamma^{\prime} \in \Gamma$ such that $\gamma^{\prime} \circ \tilde{f}=\tilde{f} \circ \gamma$. This correspondence $\gamma \rightarrow \gamma^{\prime}$ is determined by the reference $\tilde{f}$, and is obviously a homomorphism.

Definition 3. The endomorphism $\tilde{f}_{*}: \Gamma \rightarrow \Gamma$ determined by the lifting $\tilde{f}$ of $f$ is defined by

$$
\tilde{f}_{*}(\gamma) \circ \tilde{f}=\tilde{f} \circ \gamma .
$$

It is well known that $\Gamma \cong \pi_{1}(X)$. We shall identify $\pi=\pi_{1}\left(X, x_{0}\right)$ and $\Gamma$ in the following way. Pick base points $x_{0} \in X$ and $\tilde{x}_{0} \in p^{-1}\left(x_{0}\right) \subset \tilde{X}$ once for all. Now points of $\tilde{X}$ are in 1-1 correspondence with homotopy classes of paths in $X$ which start at $x_{0}$ : for $\tilde{x} \in \tilde{X}$ take any path in $\tilde{X}$ from $\tilde{x}_{0}$ to $\tilde{x}$ and project it onto $X$; conversely for a path $c$ starting at $x_{0}$, lift it to a path in $\tilde{X}$ which starts at $\tilde{x}_{0}$, and then take its endpoint. In 
this way, we identify a point of $\tilde{X}$ with a path class $\langle c\rangle$ in $X$ starting from $x_{0}$. Under this identification, $\tilde{x}_{0}=\langle e\rangle$ is the unit element in $\pi_{1}\left(X, x_{0}\right)$. The action of the loop class $\alpha=\langle a\rangle \in \pi_{1}\left(X, x_{0}\right)$ on $\tilde{X}$ is then given by

$$
\alpha=\langle a\rangle:\langle c\rangle \rightarrow \alpha . c=\langle a . c\rangle .
$$

Now we have the following relationship between $\tilde{f}_{*}: \pi \rightarrow \pi$ and

$$
f_{*}: \pi_{1}\left(X, x_{0}\right) \rightarrow \pi_{1}\left(X, f\left(x_{0}\right)\right) .
$$

Lemma 5. Suppose $\tilde{f}\left(\tilde{x}_{0}\right)=\langle w\rangle$. Then the following diagram commutes:

$$
\pi_{1}\left(X, x_{0}\right) \stackrel{f_{*}}{\longrightarrow} \pi_{1}\left(X, f\left(x_{0}\right)\right)
$$

where $w_{*}$ is the isomorphism induced by the path $w$.

In other words, for every $\alpha=\langle a\rangle \in \pi_{1}\left(X, x_{0}\right)$, we have $\tilde{f}_{*}(\langle a\rangle)=\left\langle w(f \circ a) w^{-1}\right\rangle$.

Remark 3. In particular, if $x_{0} \in p\left(\operatorname{Fix}(\tilde{f})\right.$ and $\tilde{x}_{0} \in \operatorname{Fix}(\tilde{f})$, then $\tilde{f}_{*}=f_{*}$.

LEMMA 6. Lifting classes of $f$ are in 1-1 correspondence with $\tilde{f}_{*}$-conjugacy classes in $\pi$, the lifting class $[\gamma \circ \tilde{f}]$ corresponding to the $\tilde{f}_{*}$-conjugacy class of $\gamma$. We therefore have $R(f)=R\left(\tilde{f}_{*}\right)$.

We shall say that the fixed point class $p(\operatorname{Fix}(\gamma \circ \tilde{f}))$, which is labeled with the lifting class $[\gamma \circ \tilde{f}]$, corresponds to the $\tilde{f}_{*}$-conjugacy class of $\gamma$. Thus $\tilde{f}_{*}$-conjugacy classes in $\pi$ serve as coordinates for fixed point classes of $f$, once a reference lifting $\tilde{f}$ is chosen.

Let us consider a homomorphism from $\pi$ sending an $\tilde{f}_{*}$-conjugacy class to one element:

Lemma 7 ([28]). The composition $\eta \circ \theta$,

$$
\pi=\pi_{1}\left(X, x_{0}\right) \stackrel{\theta}{\longrightarrow} H_{1}(X) \stackrel{\eta}{\longrightarrow} \operatorname{Coker}\left[H_{1}(X) \stackrel{1-f_{1 *}}{\longrightarrow} H_{1}(X)\right],
$$

where $\theta$ is abelianization and $\eta$ is the natural projection, sends every $\tilde{f}_{*}$-conjugacy class to a single element. Moreover, any group homomorphism $\zeta: \pi \rightarrow G$ which sends every $\tilde{f}_{*}$-conjugacy class to a single element factors through $\eta \circ \theta$.

Definition 4. A map $f: X \rightarrow X$ is said to be eventually commutative if there exists a natural number $n$ such that $f_{*}^{n}\left(\pi_{1}\left(X, x_{0}\right)\right) \quad\left(\subset \pi_{1}\left(X, f^{n}\left(x_{0}\right)\right)\right)$ is commutative.

By means of lemma 5 , it is easily seen that $f$ is eventually commutative iff $\tilde{f}_{*}$ is eventually commutative (see [28]).

Now using lemma 6 we may apply all theorems of $\S 1$ to the Reidemeister numbers of continuous maps.

3.1. Trace formulas and Serre bundles. For example, let us consider a topological counterpart of theorem 7 .

Let $p: E \rightarrow B$ be a Serre bundle in which $E, B$ and every fibre are connected, compact polyhedra and $F_{b}=p^{-1}(b)$ is a fibre over $b \in B$. A Serre bundle $p: E \rightarrow B$ is said to be (homotopically) orientable if for any two paths $w, w^{\prime}$ in $B$ with the same endpoints $w(0)=w^{\prime}(0)$ and $w(1)=w^{\prime}(1)$, the fibre translations $\tau_{w}, \tau_{w^{\prime}}: F_{w(0)} \rightarrow F_{w(1)}$ are homotopic. A map $f: E \rightarrow E$ is called a fibre map if there is an induced map 
$\bar{f}: B \rightarrow B$ such that $p \circ f=\bar{f} \circ p$. Let $p: E \rightarrow B$ be an orientable Serre bundle and let $f: E \rightarrow E$ be a fibre map. Then for any two fixed points $b, b^{\prime}$ of $\bar{f}: B \rightarrow B$ the maps $f_{b}=\left.f\right|_{F_{b}}$ and $f_{b^{\prime}}=\left.f\right|_{F_{b^{\prime}}}$ have the same homotopy type; hence they have the same Reidemeister numbers $R\left(f_{b}\right)=R\left(f_{b^{\prime}}\right)[28]$.

THeOREM 8. Suppose that $f: E \rightarrow E$ admits a Fadell splitting in the sense that for some $e$ in $\operatorname{Fix} f$ and $b=p(e)$ the following conditions are satisfied:

1. the sequence

$$
0 \rightarrow \pi_{1}\left(F_{b}, e\right) \stackrel{i_{*}}{\longrightarrow} \pi_{1}(E, e) \stackrel{p_{*}}{\longrightarrow} \pi_{1}(B, e) \rightarrow 0
$$

is exact,

2. $p_{*}$ admits a right inverse (section) $\sigma$ such that $\operatorname{Im} \sigma$ is a normal subgroup of $\pi_{1}(E, e)$ and $f_{*}(\operatorname{Im} \sigma) \subset \operatorname{Im} \sigma$.

If we have trace formulas of the type (8) for $R(\bar{f})$ and $R\left(f_{b}\right)$ then we have a trace formula of the same type for $R(f)$.

\section{Trace formulas for the Nielsen numbers}

4.1. The Jiang subgroup and trace formula. From the homotopy invariance theorem (see [28]) it follows that if a homotopy $\left\{h_{t}\right\}: f \cong g: X \rightarrow X$ lifts to a homotopy $\left\{\tilde{h}_{t}\right\}: \tilde{f} \cong \tilde{g}: \tilde{X} \rightarrow \tilde{X}$, then we have $\operatorname{Index}(f, p(\operatorname{Fix} \tilde{f}))=\operatorname{Index}(g, p(\operatorname{Fix} \tilde{g}))$. Suppose $\left\{h_{t}\right\}$ is a cyclic homotopy $\left\{h_{t}\right\}: f \cong f$; then this lifts to a homotopy from a given lifting $\tilde{f}$ to another lifting $\tilde{f}^{\prime}=\alpha \circ \tilde{f}$, and we have

$$
\operatorname{Index}(f, p(\operatorname{Fix} \tilde{f}))=\operatorname{Index}(f, p(\operatorname{Fix} \alpha \circ \tilde{f})) .
$$

In other words, a cyclic homotopy induces a permutation of lifting classes (and hence of fixed point classes); those in the same orbit of this permutation have the same index. This idea is applied to the computation of $N(f)$.

DEFINITION 5. The trace subgroup of cyclic homotopies (the Jiang subgroup) $I(\tilde{f}) \subset \pi$ is defined by

$$
I(\tilde{f})=\left\{\begin{array}{l|l}
\alpha \in \pi & \begin{array}{l}
\text { there exists a cyclic homotopy } \\
\left\{h_{t}\right\}: f \cong f \text { which lifts to } \\
\left\{\tilde{h}_{t}\right\}: \tilde{f} \cong \alpha \circ \tilde{f}
\end{array}
\end{array}\right\}
$$

(see $[28])$.

Let $Z(G)$ denote the centre of a group $G$, and let $Z(K, G)$ denote the centralizer of the subgroup $K \subset G$. The Jiang subgroup has the following properties: $1 . I(\tilde{f}) \subset Z\left(\tilde{f}_{*}(\pi), \pi\right)$; 2. $I\left(\operatorname{id}_{\tilde{X}}\right) \subset Z(\pi) ; 3 . I(\tilde{g}) \subset I(\tilde{g} \circ \tilde{f}) ; 4 . \tilde{g}_{*}(I(\tilde{f})) \subset I(\tilde{g} \circ \tilde{f}) ; 5 . I\left(\operatorname{id}_{\tilde{X}}\right) \subset I(\tilde{f})$.

The class of path-connected spaces $X$ satisfying the condition $I\left(\operatorname{id}_{\tilde{X}}\right)=\pi=\pi_{1}\left(X, x_{0}\right)$ is closed under homotopy equivalence and the topological product operation, and contains the simply connected spaces, generalized lens spaces, $H$-spaces and homogeneous spaces of the form $G / G_{0}$ where $G$ is a topological group and $G_{0}$ a subgroup which is a connected, compact Lie group (for the proofs see [28]).

From theorem 1 and results of Jiang [28] it follows: 
THEOREM 9. Suppose that there is an integer $m$ such that $\tilde{f}_{*}^{m}(\pi) \subset I\left(\tilde{f}^{m}\right)$ and $L(f) \neq$ 0. Then

$$
N(f)=R(f)=(-1)^{r+p} \sum_{i=0}^{k}(-1)^{i} \operatorname{Tr}\left(\Lambda^{i} f_{1 *}^{\infty} \otimes A\right) .
$$

where $k$ is $\operatorname{rg} H_{1}(X, \mathbb{Z})^{\infty}, A$ is linear map on the complex vector space of complex valued functions on the group Tors $H_{1}(X, \mathbb{Z})$, $p$ the number of $\mu \in \operatorname{Spec} f_{1 *}^{\infty}$ such that $\mu<-1$, and $r$ the number of real eigenvalues of $f_{1 *}^{\infty}$ whose absolute value is $>1$.

Proof. We have $\tilde{f}_{*}(\pi) \subset I(\tilde{f})($ see $[28])$. For any $\alpha \in \pi, p(\operatorname{Fix} \alpha \circ \tilde{f})=p\left(\operatorname{Fix} \tilde{f}_{*}(\alpha) \circ \tilde{f}\right)$ by the fact (see [28]) that $\alpha$ and $\tilde{f}_{*}(\alpha)$ are in the same $\tilde{f}_{*}$-conjugacy class.

Since $\tilde{f}_{*}(\pi) \subset I(\tilde{f})$, there is a homotopy $\left\{h_{t}\right\}: f \cong f$ which lifts to $\left\{\tilde{h}_{t}\right\}: \tilde{f} \cong \tilde{f}_{*}(\alpha) \circ \tilde{f}$. Hence $\operatorname{Index}(f, p(\operatorname{Fix} \tilde{f}))=\operatorname{Index}(f, p($ Fix $\alpha \circ \tilde{f}))$. Since $\alpha \in \pi$ is arbitrary, any two fixed point classes of $f$ have the same index. It immediately follows that $L(f)=0$ implies $N(f)=0$ and $L(f) \neq 0$ implies $N(f)=R(f)$. By property $1, \tilde{f}(\pi) \subset I(\tilde{f}) \subset Z\left(\tilde{f}_{*}(\pi), \pi\right)$, so $\tilde{f}_{*}(\pi)$ is Abelian. Hence $\tilde{f}_{*}$ is eventually commutative and $N(f)=R(f)=R\left(\tilde{f}_{*}\right)=$ $R\left(f_{1 *}\right)$. The result now follows from theorem 1 .

ExAmple 1. Let $f: X \rightarrow X$ be a hyperbolic endomorphism of the torus $T^{k}$. Then $H_{1}(X, \mathbb{Z})$ is torsion free and

$$
N(f)=R(f)=(-1)^{r+p} \sum_{i=0}^{k}(-1)^{i} \operatorname{Tr}\left(\Lambda^{i} f_{1 *}\right) .
$$

4.2. Polyhedra with finite fundamental group. For a compact polyhedron $X$ with finite fundamental group, $\pi_{1}(X)$, the universal cover $\tilde{X}$ is compact, so we may explore the relation between $L(\tilde{f})$ and $\operatorname{Index}(p(\operatorname{Fix} \tilde{f}))$.

Definition 6 . The number $\mu([\tilde{f}])=\#$ Fix $\tilde{f}_{*}$, defined to be the order of the finite group Fix $\tilde{f}_{*}$, is called the multiplicity of the lifting class $[\tilde{f}]$, or of the fixed point class $p(\operatorname{Fix} \tilde{f})$.

LEMMA 8 ([28]).

$$
L(\tilde{f})=\mu([\tilde{f}]) \cdot \operatorname{Index}(f, p(\operatorname{Fix} \tilde{f})) .
$$

Let $W$ be the complex vector space of complex valued class functions on the fundamental group $\pi$. The map $\tilde{f}_{*}$ induces a linear map $B: W \rightarrow W$ defined by

$$
B(f):=f \circ \tilde{f}_{*} .
$$

THEOREM 10. Let $X$ be a connected, compact polyhedron with finite fundamental group $\pi$. Suppose that the action of $\pi$ on the rational homology of the universal cover $\tilde{X}$ is trival, i.e. for every covering translation $\alpha \in \pi, \alpha_{*}=\mathrm{id}: H_{*}(\tilde{X}, \mathbb{Q}) \rightarrow H_{*}(\tilde{X}, \mathbb{Q})$. Let $L(f) \neq 0$. Then

$$
N(f)=R(f)=\operatorname{Tr} B .
$$

Proof. Under our assumption on $X$, any two liftings $\tilde{f}$ and $\alpha \circ \tilde{f}$ induce the same homology homomorphism $H_{*}(\tilde{X}, \mathbb{Q}) \rightarrow H_{*}(\tilde{X}, \mathbb{Q})$, and have thus the same value of $L(\tilde{f})$. From lemma 8 it follows that any two fixed point classes $f$ are either both essential or both inessential. Since $L(f) \neq 0$ there is at least one essential fixed point class of $f$. 
Therefore all fixed point classes of $f$ are essential and $N(f)=R(f)$. The formula for $N(f)$ follows now from theorem 4 .

Lemma 9. Let $X$ be a polyhedron with finite fundamental group $\pi$ and let $p: \tilde{X} \rightarrow X$ be its universal covering. Then the action of $\pi$ on the rational homology of $\tilde{X}$ is trivial iff $H_{*}(\tilde{X} ; \mathbb{Q}) \cong H_{*}(X ; \mathbb{Q})$.

COROLlary 3. Let $\tilde{X}$ be a compact 1 -connected polyhedron which is a rational homology $n$-sphere, where $n$ is odd. Let $\pi$ be a finite group acting freely on $\tilde{X}$ and let $X=\tilde{X} / \pi$. Then theorem 10 applies.

Proof. The projection $p: \tilde{X} \rightarrow X=\tilde{X} / \pi$ is a universal covering space of $X$. For every $\alpha \in \pi$, the degree of $\alpha: \tilde{X} \rightarrow \tilde{X}$ must be 1 , because $L(\alpha)=0$ ( $\alpha$ has no fixed points). Hence $\alpha_{*}=\mathrm{id}: H_{*}(\tilde{X} ; \mathbb{Q}) \rightarrow H_{*}(\tilde{X} ; \mathbb{Q})$.

Corollary 4. If $X$ is a closed 3-manifold with finite $\pi$, then theorem 10 applies.

Proof. $\tilde{X}$ is an orientable, simply connected manifold, hence a homology 3 -sphere. We apply corollary 3 .

Corollary 5. Let $X=L\left(m, q_{1}, \ldots, q_{r}\right)$ be a generalized lens space and $f: X \rightarrow X$ a continuous map with $f_{1 *}(1)=d$ where $d \neq 1$. Then theorem 10 applies.

Proof. By corollary 1 we see that theorem 10 applies for lens spaces. Since $\pi_{1}(X)=$ $\mathbb{Z} / m \mathbb{Z}$, the map $f$ is eventually commutative. A lens space has the structure of a $\mathrm{CW}$ complex with one cell $e_{i}$ in each dimension $0 \leq i \leq 2 l+1$. The boundary map is given by $\partial e_{2 k}=m \cdot e_{2 k-1}$ for even cells, and $\partial e_{2 k+1}=0$ for odd cells. From this we may calculate the Lefschetz numbers: $L(f)=1-d^{(l+1)} \neq 0$.

\subsection{Other special cases}

\subsubsection{Self-map of a nilmanifold. Theorem 6 implies}

THEOREM 11. Let $f$ be any continuous map of a nilmanifold $M$ to itself. If $R(f)$ is finite then

$$
N(f)=R(f)=(-1)^{r+p} \sum_{i=0}^{m}(-1)^{i} \operatorname{Tr} \Lambda^{i} \tilde{F},
$$

where $\tilde{F}, m, r$, and $p$ are the same as in theorem 6 .

4.3.2. Pseudo-Anosov homeomorphisms of a compact surface. Let $X$ be a compact surface of negative Euler characteristic and $f: X \rightarrow X$ is a pseudo-Anosov homeomorphism, i.e. there is a number $\lambda>1$ and a pair of transverse measured foliations $\left(F^{s}, \mu^{s}\right)$ and $\left(F^{u}, \mu^{u}\right)$ such that $f\left(F^{s}, \mu^{s}\right)=\left(F^{s}, \frac{1}{\lambda} \mu^{s}\right)$ and $f\left(F^{u}, \mu^{u}\right)=\left(F^{u}, \lambda \mu^{u}\right)$. Fathi and Shub [10] has proved the existence of Markov partitions for a pseudo-Anosov homeomorphism. The existence of Markov partitions implies that there is a symbolic dynamics for $(X, f)$. This means that there is a finite set $N$, a matrix $A=\left(a_{i j}\right)_{(i, j) \in N \times N}$ with entries 0 or 1 and a surjective map $p: \Omega \rightarrow X$, where

$$
\Omega=\left\{\left(x_{n}\right)_{n \in \mathbb{Z}}: a_{x_{n} x_{n+1}}=1, n \in \mathbb{Z}\right\}
$$


such that $p \circ \sigma=f \circ p$ where $\sigma$ is the shift (to the left) of the sequence $\left(x_{n}\right)$ of symbols. We have first [5]:

$$
\text { \# Fix } \sigma^{n}=\operatorname{Tr} A^{n} \text {. }
$$

In general $p$ is not bijective. The non-injectivity of $p$ is due to the fact that the rectangles of the Markov partition can meet on their boundaries. To cancel the overcounting of periodic points on these boundaries, we use Manning's combinatorial arguments [34] proposed in the case of Axiom A diffeomorphisms (see also [39]). Namely, we construct finitely many subshifts of finite type $\sigma_{i}, i=0,1, \ldots, m$, such that $\sigma_{0}=\sigma$, the other shifts semi-conjugate with restrictions of $f[39]$, and signs $\epsilon_{i} \in\{-1,1\}$ such that for each $n$

$$
\text { \# Fix } f^{n}=\sum_{i=0}^{m} \epsilon_{i} \cdot \# \operatorname{Fix} \sigma_{i}^{n}=\sum_{i=0}^{m} \epsilon_{i} \cdot \operatorname{Tr} A_{i}^{n},
$$

where $A_{i}$ is the transition matrix corresponding to the subshift of finite type $\sigma_{i}$. For a pseudo-Anosov homeomorphism of a compact surface $N\left(f^{n}\right)=\# \operatorname{Fix}\left(f^{n}\right)$ for each $n>0$ [47]. So we have following trace formula for Nielsen numbers:

THEOREM 12. Let $X$ be a compact surface of negative Euler characteristic and $f$ : $X \rightarrow X$ a pseudo-Anosov homeomorphism. Then

$$
N\left(f^{n}\right)=\sum_{i=0}^{m} \epsilon_{i} \cdot \operatorname{Tr} A_{i}^{n}
$$

\subsubsection{Homeomorphisms of hyperbolic 3-manifolds}

TheOREM 13 ([30]). Suppose $M$ is an orientable compact connected 3-manifold such that int $M$ admits a complete hyperbolic structure with finite volume and $f: M \rightarrow M$ is an orientation preserving homeomorphism. Then

$$
N(f)=L(f)=\sum_{k=0}^{\operatorname{dim} M}(-1)^{k} \operatorname{Tr}\left[f_{* k}: H_{k}(X ; \mathbb{Q}) \rightarrow H_{k}(X ; \mathbb{Q})\right] .
$$

5. The Reidemeister trace formula for generalized Lefschetz numbers. The results of this section are well known (see [41], [48], [9], [29]). We shall use them later to estimate the radius of convergence of the Nielsen zeta function. The fundamental group $\pi=\pi_{1}\left(X, x_{0}\right)$ splits into $\tilde{f}_{*}$-conjugacy classes. Let $\pi_{f}$ denote the set of $\tilde{f}_{*}$-conjugacy classes, and $\mathbb{Z} \pi_{f}$ denote the Abelian group freely generated by $\pi_{f}$. We will use the bracket notation $a \rightarrow[a]$ for both projections $\pi \rightarrow \pi_{f}$ and $\mathbb{Z} \pi \rightarrow \mathbb{Z} \pi_{f}$. Let $x$ be a fixed point of $f$. Take a path $c$ from $x_{0}$ to $x$. The $\tilde{f}_{*}$-conjugacy class in $\pi$ of the loop $c \cdot(f \circ c)^{-1}$, which is evidently independent of the choice of $c$, is called the coordinate of $x$. Two fixed points are in the same fixed point class $F$ iff they have the same coordinates. This $\tilde{f}_{*}$-conjugacy class is thus called the coordinate of the fixed point class $F$ and denoted $\operatorname{cd}_{\pi}(F, f)$ (compare with description in section 2). The generalized Lefschetz number or the Reidemeister trace [41] is defined as

$$
L_{\pi}(f):=\sum_{F} \operatorname{ind}(F, f) \cdot \operatorname{cd}_{\pi}(F, f) \in \mathbb{Z} \pi_{f}
$$


the summation being over all essential fixed point classes $F$ of $f$. The Nielsen number $N(f)$ is the number of non-zero terms in $L_{\pi}(f)$, and the indices of the essential fixed point classes appear as the coefficients in $L_{\pi}(f)$. This invariant used to be called the Reidemeister trace because it can be computed as an alternating sum of traces on the chain level as follows ([41], [48]). Assume that $X$ is a finite cell complex and $f: X \rightarrow X$ is a cellular map. A cellular decomposition $e_{j}^{d}$ of $X$ lifts to a $\pi$-invariant cellular structure on the universal covering $\tilde{X}$. Choose an arbitrary lift $\tilde{e}_{j}^{d}$ for each $e_{j}^{d}$. They constitute a free $\mathbb{Z} \pi$-basis for the cellular chain complex of $\tilde{X}$. The lift $\tilde{f}$ of $f$ is also a cellular map. In every dimension $d$, the cellular chain map $\tilde{f}$ gives rise to a $\mathbb{Z} \pi$-matrix $\tilde{F}_{d}$ with respect to the above basis, i.e. $\tilde{F}_{d}=\left(a_{i j}\right)$ if $\tilde{f}\left(\tilde{e}_{i}^{d}\right)=\sum_{j} a_{i j} \tilde{e}_{j}^{d}$, where $a_{i j} \in \mathbb{Z} \pi$. Then we have the Reidemeister trace formula

$$
L_{\pi}(f)=\sum_{d}(-1)^{d}\left[\operatorname{Tr} \tilde{F}_{d}\right] \in \mathbb{Z} \pi_{f}
$$

5.1. The mapping torus approach to the Reidemeister trace formula. Now we describe an alternative approach to the Reidemeister trace formula proposed recently by Jiang [29]. This approach is useful when we study the periodic points of $f$, i.e. the fixed points of the iterates of $f$.

The mapping torus $T_{f}$ of $f: X \rightarrow X$ is the space obtained from $X \times[0, \infty)$ by identifying $(x, s+1)$ with $(f(x), s)$ for all $x \in X, s \in[0, \infty)$. On $T_{f}$ there is a natural semiflow $\phi: T_{f} \times[0, \infty) \rightarrow T_{f}, \phi_{t}(x, s)=(x, s+t)$ for all $t \geq 0$. Then the map $f: X \rightarrow X$ is the return map of the semi-flow $\phi$. A point $x \in X$ and a positive number $\tau>0$ determine the orbit curve $\phi_{(x, \tau)}:=\phi_{t}(x)_{0 \leq t \leq \tau}$ in $T_{f}$. Take the base point $x_{0}$ of $X$ as the base point of $T_{f}$. It is known that the fundamental group $H:=\pi_{1}\left(T_{f}, x_{0}\right)$ is obtained from $\pi$ by adding a new generator $z$ and adding the relations $z^{-1} g z=\tilde{f}_{*}(g)$ for all $g \in \pi=\pi_{1}\left(X, x_{0}\right)$. Let $H_{c}$ denote the set of conjugacy classes in $H$. Let $\mathbb{Z} H$ be the integral group ring of $H$, and let $\mathbb{Z} H_{c}$ be the free Abelian group with basis $H_{c}$. We again use the bracket notation $a \rightarrow[a]$ for both projections $H \rightarrow H_{c}$ and $\mathbb{Z} H \rightarrow \mathbb{Z} H_{c}$. If $F^{n}$ is a fixed point class of $f^{n}$, then $f\left(F^{n}\right)$ is also fixed point class of $f^{n}$ and $\operatorname{ind}\left(f\left(F^{n}\right), f^{n}\right)=\operatorname{ind}\left(F^{n}, f^{n}\right)$. Thus $f$ acts as an index-preserving permutation among fixed point classes of $f^{n}$. By definition, an $n$-orbit class $O^{n}$ of $f$ is the union of elements of an orbit of this action. In other words, two points $x, x^{\prime} \in \operatorname{Fix}\left(f^{n}\right)$ are said to be in the same $n$-orbit class of $f$ if and only if some $f^{i}(x)$ and some $f^{j}\left(x^{\prime}\right)$ are in the same fixed point class of $f^{n}$. The set $\operatorname{Fix}\left(f^{n}\right)$ splits into a disjoint union of $n$-orbit classes. A point $x$ is a fixed point of $f^{n}$ or a periodic point of period $n$ if and only if orbit curve $\phi_{(x, n)}$ is a closed curve. The free homotopy class of the closed curve $\phi_{(x, n)}$ will be called the $H$-coordinate of $x$, written $\operatorname{cd}_{H}(x, n)=\left[\phi_{(x, n)}\right] \in H_{c}$. It follows that periodic points $x$ of period $n$ and $x^{\prime}$ of period $n^{\prime}$ have the same $H$-coordinate if and only if $n=n^{\prime}$ and $x, x^{\prime}$ belong to the same $n$-orbit class of $f$. Thus it is possible equivalently to define $x, x^{\prime} \in \operatorname{Fix} f^{n}$ to be in the same $n$-orbit class if and only if they have the same $H$-coordinate. Recently, Jiang [29] has considered a generalized Lefschetz number with respect to $H$,

$$
L_{H}\left(f^{n}\right):=\sum_{O^{n}} \operatorname{ind}\left(O^{n}, f^{n}\right) \cdot \operatorname{cd}_{H}\left(O^{n}\right) \in \mathbb{Z} H_{c},
$$


and proved the following trace formula:

$$
L_{H}\left(f^{n}\right)=\sum_{d}(-1)^{d}\left[\operatorname{Tr}\left(z \tilde{F}_{d}\right)^{n}\right] \in \mathbb{Z} H_{c},
$$

where $\tilde{F}_{d}$ are the $\mathbb{Z} \pi$-matrices defined in (16) and $z \tilde{F}_{d}$ is regarded as a $\mathbb{Z} H$-matrix.

\section{The Reidemeister zeta function of a group endomorphism}

Problem. For which groups and endomorphisms is the Reidemeister zeta function a rational function? When does it have a functional equation? Is $R_{\phi}(z)$ an algebraic function?

6.1. Reidemeister zeta functions of eventually commutative endomorphisms. As we remarked in section 1 to find out about the Reidemeister zeta functions of eventually commutative endomorphisms, it is sufficient to study the zeta functions of endomorphisms of Abelian groups.

THEOREM 14. Let $G$ be a finitely generated Abelian group and $\phi$ an endomorphism of $G$. Then $R_{\phi}(z)$ is a rational function and is equal to

$$
R_{\phi}(z)=\left(\prod_{i=0}^{k} \operatorname{det}\left(1-\Lambda^{i} \phi^{\infty} \otimes A \cdot \sigma \cdot z\right)^{(-1)^{i+1}}\right)^{(-1)^{r}}
$$

where the matrix $A$ is defined in lemma 3, $\sigma=(-1)^{p}, p, r$ and $k$ are constants described in theorem 1.

Proof. If we repeat the proof of theorem 1 for $\phi^{n}$ instead $\phi$ we obtain $R\left(\phi^{n}\right)=$ $R\left(\left(\phi^{\infty}\right)^{n} \cdot R\left(\left(\phi^{\text {finite }}\right)^{n}\right)\right.$. From this and lemmas 2 and 3 we have the trace formula for $R\left(\phi^{n}\right)$ :

$$
\begin{aligned}
R\left(\phi^{n}\right) & =(-1)^{r+p n} \sum_{i=0}^{k}(-1)^{i} \operatorname{Tr} \Lambda^{i}\left(\phi^{\infty}\right)^{n} \cdot \operatorname{Tr} A^{n} \\
& =(-1)^{r+p n} \sum_{i=0}^{k}(-1)^{i} \operatorname{Tr}\left(\Lambda^{i}\left(\phi^{\infty}\right)^{n} \otimes A^{n}\right) \\
& =(-1)^{r+p n} \sum_{i=0}^{k}(-1)^{i} \operatorname{Tr}\left(\Lambda^{i} \phi^{\infty} \otimes A\right)^{n}
\end{aligned}
$$

We now calculate directly

$$
\begin{aligned}
R_{\phi}(z) & =\exp \left(\sum_{n=1}^{\infty} \frac{R\left(\phi^{n}\right)}{n} z^{n}\right)=\exp \left(\sum_{n=1}^{\infty} \frac{(-1)^{r} \sum_{i=0}^{k}(-1)^{i} \operatorname{Tr}\left(\Lambda^{i} \phi^{\infty} \otimes A\right)^{n}}{n}(\sigma \cdot z)^{n}\right) \\
& =\left(\prod_{i=0}^{k}\left(\exp \left(\sum_{n=1}^{\infty} \frac{1}{n} \operatorname{Tr}\left(\Lambda^{i} \phi^{\infty} \otimes A\right)^{n} \cdot(\sigma \cdot z)^{n}\right)\right)^{(-1)^{i}}\right)^{(-1)^{r}} \\
& =\left(\prod_{i=0}^{k} \operatorname{det}\left(1-\Lambda^{i} \phi^{\infty} \otimes A \cdot \sigma \cdot z\right)^{(-1)^{i+1}}\right)^{(-1)^{r}} .
\end{aligned}
$$




\subsection{Endomorphisms of finite groups}

THEOREM 15. Let $\phi$ be an endomorphism of a finite group $G$. Then $R_{\phi}(z)$ is a rational function and is given by the formula

$$
R_{\phi}(z)=\frac{1}{\operatorname{det}(1-B z)}
$$

where $B$ is defined in theorem 4.

Proof. From theorem 4 it follows that $R\left(\phi^{n}\right)=\operatorname{Tr} B^{n}$ for every $n>0$. We now calculate directly

$$
\begin{aligned}
R_{\phi}(z) & =\exp \left(\sum_{n=1}^{\infty} \frac{R\left(\phi^{n}\right)}{n} z^{n}\right)=\exp \left(\sum_{n=1}^{\infty} \frac{\operatorname{Tr} B^{n}}{n} z^{n}\right)=\exp \left(\operatorname{Tr} \sum_{n=1}^{\infty} \frac{B^{n}}{n} z^{n}\right) \\
& =\exp (\operatorname{Tr}(-\log (1-B z)))=\frac{1}{\operatorname{det}(1-B z)} .
\end{aligned}
$$

6.3. Endomorphisms of the direct sum of a free Abelian and a finite group

TheOREm 16. Let $G$ be the direct sum of a free Abelian and a finite group and $\phi$ an endomorphism of $G$. If the numbers $R\left(\phi^{n}\right)$ are all finite then $R_{\phi}(z)$ is a rational function and is equal to

$$
R_{\phi}(z)=\left(\prod_{i=0}^{k} \operatorname{det}\left(1-\Lambda^{i} \phi^{\infty} \otimes B \cdot \sigma \cdot z\right)^{(-1)^{i+1}}\right)^{(-1)^{r}}
$$

where the matrix $B$ is defined in theorem $4, \sigma=(-1)^{p}, p, r$ and $k$ are constants described in theorem 5.

ProOF. From proposition 2 it follows that $R\left(\phi^{n}\right)=R\left(\left(\phi^{\infty}\right)^{n} \cdot R\left(\left(\phi^{\text {finite }}\right)^{n}\right)\right.$. From now on the proof repeats the proof of theorem 14 .

\subsection{Endomorphisms of nilpotent groups}

THEOREM 17. If $\Gamma$ is a finitely generated torsion free nilpotent group and $\phi$ an endomorphism of $\Gamma$ then $R_{\phi}(z)$ is a rational function and is equal to

$$
R_{\phi}(z)=\left(\prod_{i=0}^{m} \operatorname{det}\left(1-\Lambda^{i} \tilde{F} \cdot \sigma \cdot z\right)^{(-1)^{i+1}}\right)^{(-1)^{r}}
$$

where $\sigma=(-1)^{p}, p, r, m$ and $\tilde{F}$ are defined in section 1.5.

PROOF. If we repeat the proof of theorem 6 for $\phi^{n}$ instead $\phi$ we obtain $R\left(\phi^{n}\right)=$ $(-1)^{r+p n} \operatorname{det}(1-\tilde{F})$ (we suppose that the Reidemeister numbers $R\left(\phi^{n}\right)$ are finite for all $n)$. The last formula implies the trace formula for $R\left(\phi^{n}\right)$ :

$$
R\left(\phi^{n}\right)=(-1)^{r+p n} \sum_{i=0}^{m}(-1)^{i} \operatorname{Tr}\left(\Lambda^{i} \tilde{F}\right)^{n} .
$$

From this we have formula (23) immediately by direct calculation as in theorem 14 .

Corollary 6. Let the assumptions of theorem 17 hold. Then the poles and zeros of the Reidemeister zeta function are complex numbers which are the reciprocals of eigen- 
values of one of the matrices

$$
\Lambda^{i}(\tilde{F}): \Lambda^{i}(\tilde{G}) \longrightarrow \Lambda^{i}(\tilde{G}), \quad 0 \leq i \leq \operatorname{rank} \Gamma .
$$

\subsubsection{Functional equation}

THEOREM 18. Let $\phi: \Gamma \rightarrow \Gamma$ be an endomorphism of a finitely generated torsion free nilpotent group $\Gamma$. Then the Reidemeister zeta function $R_{\phi}(z)$ has the following functional equation:

$$
R_{\phi}\left(\frac{1}{d z}\right)=\epsilon_{2} \cdot R_{\phi}(z)^{(-1)^{\operatorname{Rank} \Gamma}} .
$$

where $d=\operatorname{det} \tilde{F}$ and $\epsilon_{1}$ are constants in $\mathbb{C}$.

Proof. Via the natural nonsingular pairing $\left(\Lambda^{i} \tilde{F}\right) \otimes\left(\Lambda^{m-i} \tilde{F}\right) \rightarrow \mathbb{C}$ the operators $\Lambda^{m-i} \tilde{F}$ and $d \cdot\left(\Lambda^{i} \tilde{F}\right)^{-1}$ are adjoint to each other.

We consider an eigenvalue $\lambda$ of $\Lambda^{i} \tilde{F}$. By theorem 17, this contributes a term

$$
\left(\left(1-\frac{\lambda \sigma}{d z}\right)^{(-1)^{i+1}}\right)^{(-1)^{r}}
$$

to $R_{\phi}\left(\frac{1}{d z}\right)$. We rewrite this term as

$$
\left(\left(1-\frac{d \sigma z}{\lambda}\right)^{(-1)^{i+1}} \cdot\left(\frac{-d z}{\lambda \sigma}\right)^{(-1)^{i}}\right)^{(-1)^{r}}
$$

and note that $\frac{d}{\lambda}$ is an eigenvalue of $\Lambda^{m-i} \tilde{F}$. Multiplying these terms together we obtain

$$
R_{\phi}\left(\frac{1}{d z}\right)=\left(\prod_{i=1}^{m} \prod_{\lambda^{(i)} \in \operatorname{Spec} \Lambda^{i} \tilde{F}}\left(\frac{1}{\lambda^{(i)} \sigma}\right)^{(-1)^{i}}\right)^{(-1)^{r}} \times R_{\phi}(z)^{(-1)^{m}}
$$

The variable $z$ has disappeared because

$$
\sum_{i=0}^{m}(-1)^{i} \operatorname{dim} \Lambda^{i} \tilde{G}=\sum_{i=0}^{m}(-1)^{i} C_{k}^{i}=0
$$

6.5. Some conjectures for wider classes of groups. For the case of almost nilpotent groups (i.e. groups with polynomial growth, in view of Gromov's theorem [25]) we believe that some power of the Reidemeister zeta function is a rational function. We intend to prove this conjecture by identifying the Reidemeister number on the nilpotent part of the group with the number of fixed points in the direct sum of the duals of the quotients of successive terms in the central series. We then hope to show that the Reidemeister number of the whole endomorphism is the sum of the numbers of orbits of such fixed points under the action of the finite quotient group (i.e. the quotient of the whole group by the nilpotent part). The situation for groups with exponential growth is very different. There one can expect the Reidemeister number to be infinite as long as the endomorphism is injective. This can be proved in the case of surface groups due to a theorem of C. Epstein (see [8]). He proves an estimate on numbers of geodesics, which when applied to the mapping torus of a pseudo-Anosov map guarantees infinitely many loops which wrap around the mapping torus exactly once. This is equivalent (see section 4.1 ) to saying that the Reidemeister number is infinite. A rigid hyperbolic group has a finite outer automorphism group [26]. 
This implies that the Reidemeister number of some iteration of the endomorphism equals the number of usual conjugacy classes which is in this case infinite. We believe this conjecture for the following reason. Let $G$ be a group with exponential growth and let $l(g)$ be the length of an element of $G$. Then one might expect that most of the time

$$
l\left(g x \phi(g)^{-1}\right)>(1+\epsilon) l(g)
$$

for $g, x \in G$. This would imply that for fixed $x$,

$$
\#\left\{g x \phi(g)^{-1}: l\left(g x \phi(g)^{-1}\right)<N\right\}<\#\left\{g \in G: l\left(g x \phi(g)^{-1}\right)<N /(1+\epsilon)\right\} .
$$

However since the group has exponential growth one can show that this would imply

$$
\frac{\#\left\{g x \phi(g)^{-1}: l\left(g x \phi(g)^{-1}\right)<N\right\}}{\#\left\{g \in G: l\left(g x \phi(g)^{-1}\right)<N\right\}} \rightarrow 0 \quad \text { as } N \rightarrow \infty .
$$

If there were only finitely many twisted conjugacy classes then we could derive a contradiction by summing the left hand side of the above formula over a set of representatives $x$ for the classes, and observing that this sum is always equal to 1 .

\section{The Reidemeister and Nielsen zeta functions of a continuous map}

REMARK 4. Using lemma 6 we may apply all theorems of section 5 to the Reidemeister zeta functions of continuous maps.

\subsection{The Jiang subgroup and the Nielsen zeta function}

THEOREM 19. Suppose that there is an integer $m$ such that $\tilde{f}_{*}^{m}(\pi) \subset I\left(\tilde{f}^{m}\right)$. If $L\left(f^{n}\right) \neq 0$ for every $n>0$, then

$$
N_{f}(z)=R_{f}(z)=\left(\prod_{i=0}^{k} \operatorname{det}\left(1-\Lambda^{i} f_{1 *}^{\infty} \otimes A \cdot \sigma \cdot z\right)^{(-1)^{i+1}}\right)^{(-1)^{r}} .
$$

If $L\left(f^{n}\right)=0$ only for finitely many $n$, then

$$
\begin{aligned}
N_{f}(z) & =\exp (P(z)) \cdot R_{f}(z) \\
& =\exp (P(z)) \cdot\left(\prod_{i=0}^{k} \operatorname{det}\left(1-\Lambda^{i} f_{1 *}^{\infty} \otimes A \cdot \sigma \cdot z\right)^{(-1)^{i+1}}\right)^{(-1)^{r}}
\end{aligned}
$$

where $P(z)$ is a polynomial, $A, k, p, \sigma$ and $r$ are as in theorem 14 .

Proof. If $L\left(f^{n}\right) \neq 0$ for every $n>0$, then formula (19) follows from theorems 9 and 14. If $L\left(f^{n}\right)=0$, then $N\left(f^{n}\right)=0$. If $L\left(f^{n}\right) \neq 0$, then $N\left(f^{n}\right)=R\left(f^{n}\right)$ (see proof of theorem 9). So the fraction $N_{f}(z) / R_{f}(z)=\exp (P(z))$, where $P(z)$ is a polynomial whose degree is the maximal $n$ such that $L\left(f^{n}\right) \neq 0$.

Corollary 7. Let the assumptions of theorem 19 hold. Then the poles and zeros of the Nielsen zeta function are complex numbers which are the reciprocals of eigenvalues of one of the matrices $\Lambda^{i}\left(f_{1 *}^{\infty} \otimes A \cdot \sigma\right)$.

Corollary 8. Let $I\left(\mathrm{id}_{\tilde{X}}\right)=\pi$. If the assumptions of theorem 19 about Lefschetz numbers hold, then formulas (24) and (25) are valid. 
Corollary 9. Suppose that $X$ is aspherical and $f$ is eventually commutative. If the assumptions of theorem 19 about Lefschetz numbers hold, then formulas (25) and (26) are valid.

7.2. Polyhedra with finite fundamental group and Nielsen zeta function

TheOREM 20. Let $X$ be a connected, compact polyhedron with finite fundamental group $\pi$. Suppose that the action of $\pi$ on the rational homology of the universal cover $\tilde{X}$ is trival, i.e. for every covering translation $\alpha \in \pi, \alpha_{*}=\mathrm{id}: H_{*}(\tilde{X}, \mathbb{Q}) \rightarrow H_{*}(\tilde{X}, \mathbb{Q})$. If $L\left(f^{n}\right) \neq 0$ for every $n>0$, then

$$
N_{f}(z)=R_{f}(z)=\frac{1}{\operatorname{det}(1-B z)} .
$$

If $L\left(f^{n}\right)=0$ only for finitely many $n$, then

$$
N_{f}(z)=\exp (P(z)) \cdot R_{f}(z)=\exp (P(z)) \cdot \frac{1}{\operatorname{det}(1-B z)},
$$

where $P(z)$ is a polynomial, $B$ is defined in theorem 4.

Proof. If $L\left(f^{n}\right) \neq 0$ for every $n>0$, then formula (27) follows from theorems 10 and 15. If $L\left(f^{n}\right)=0$, then $N\left(f^{n}\right)=0$. If $L\left(f^{n}\right) \neq 0$, then $N\left(f^{n}\right)=R\left(f^{n}\right)$ (see proof of theorem 10). So the fraction $N_{f}(z) / R_{f}(z)=\exp (P(z))$, where $P(z)$ is a polynomial whose degree is the maximal $n$ such that $L\left(f^{n}\right) \neq 0$.

COROLlary 10. Let $\tilde{X}$ be a compact 1-connected polyhedron which is a rational homology $n$-sphere, where $n$ is odd. Let $\pi$ be a finite group acting freely on $\tilde{X}$ and let $X=\tilde{X} / \pi$. Then theorem 20 applies.

Corollary 11. If $X$ is a closed 3-manifold with finite $\pi$, then theorem 20 applies.

ExAmple 2 ([3]). Let $f: S^{2} \vee S^{4} \rightarrow S^{2} \vee S^{4}$ be a continuous map of the bouquet of spheres such that the restriction $\left.f\right|_{S^{4}}=\mathrm{id}_{S^{4}}$ and the degree of the restriction $\left.f\right|_{S^{2}}$ : $S^{2} \rightarrow S^{2}$ is -2 . Then $L(f)=0$, hence $N(f)=0$ since $S^{2} \vee S^{4}$ is simply connected. For $k>1$ we have $L\left(f^{k}\right)=2+(-2)^{k} \neq 0$, therefore $N\left(f^{k}\right)=1$. From this we have by direct calculation that

$$
N_{f}(z)=\exp (-z) \cdot \frac{1}{1-z} .
$$

REMARK 5. We remark that in all known cases the Nielsen zeta function is a nice function. By this we mean that it is a product of an exponential of a polynomial with a function some power of which is rational. Maybe this is a general pattern; it could however be argued that this just reflects our inability to calculate the Nielsen numbers in the general case.

7.3. Nielsen zeta function in other special cases. Theorem 17 implies

THEOREM 21. Let $f$ be any continuous map of a nilmanifold $M$ to itself. If $R\left(f^{n}\right)$ is finite for all $n$ then

$$
N_{f}(z)=R_{f}(z)=\left(\prod_{i=0}^{m} \operatorname{det}\left(1-\Lambda^{i} \tilde{F} \cdot \sigma \cdot z\right)^{(-1)^{i+1}}\right)^{(-1)^{r}}
$$

where $\sigma=(-1)^{p}, p, r, m$ and $\tilde{F}$ are defined in section 5.4. 
Theorem 12 implies

THEOREM 22. Let $X$ be a compact surface of negative Euler characteristic and $f$ : $X \rightarrow X$ a pseudo-Anosov homeomorphism. Then

$$
N_{f}(z)=\prod_{i=0}^{m} \operatorname{det}\left(1-A_{i} \cdot z\right)^{-\epsilon_{i}}
$$

where $A_{i}$ and $\epsilon_{i}$ are the same as in theorem 12.

Theorem 13 implies

TheOrem 23. Suppose $M$ is an orientable compact connected 3-manifold such that $\operatorname{int} M$ admits a complete hyperbolic structure with finite volume and $f: M \rightarrow M$ is an orientation preserving homeomorphism. Then the Nielsen zeta function is rational and $N_{f}(z)=L_{f}(z)$.

7.4. Radius of convergence of the Nielsen zeta function. We denote by $R$ the radius of convergence of the Nielsen zeta function $N_{f}(z)$, and by $h(f)$ the topological entropy of the continuous map $f$. Let $h=\inf \{h(g) \mid g$ has the same homotopy type as $f\}$.

TheOREM 24 ([19]). For any continuous map $f$ of any compact polyhedron $X$ into itself the Nielsen zeta function has positive radius of convergence $R$ and

$$
R \geq \exp (-h)>0 .
$$

In this section we propose another proof of positivity of $R$ and give an exact algebraic lower estimate for the radius $R$ using trace formulas (16) and (18) for generalized Lefschetz numbers.

For any set $S$ let $\mathbb{Z} S$ denote the free Abelian group with the specified basis $S$. The norm in $\mathbb{Z} S$ is defined by

$$
\left\|\sum_{i} k_{i} s_{i}\right\|:=\sum_{i}\left|k_{i}\right| \in \mathbb{Z}
$$

when the $s_{i}$ in $S$ are all different.

For a $\mathbb{Z} H$-matrix $A=\left(a_{i j}\right)$, define its norm by $\|A\|:=\sum_{i, j}\left\|a_{i j}\right\|$. Then we have the inequalities $\|A B\| \leq\|A\| \cdot\|B\|$ when $A, B$ can be multiplied, and $\|\operatorname{Tr} A\| \leq\|A\|$ when $A$ is a square matrix. For a matrix $A=\left(a_{i j}\right)$ in $\mathbb{Z} S$, its matrix of norms is defined to be the matrix $A^{\text {norm }}:=\left(\left\|a_{i j}\right\|\right)$ which is a matrix of non-negative integers. In what follows, the set $S$ will be $\pi, H$ or $H_{c}$. We denote by $s(A)$ the spectral radius of $A$, $s(A)=\lim _{n}\left(\left\|A^{n}\right\| \mid\right)^{\frac{1}{n}}$, which coincides with the largest modulus of an eigenvalue of $A$.

TheOREM 25. For any continuous map $f$ of any compact polyhedron $X$ into itself the Nielsen zeta function has positive radius of convergence $R$, which admits the estimates

$$
R \geq \frac{1}{\max _{d}\left\|z \tilde{F}_{d}\right\|}>0
$$

and

$$
R \geq \frac{1}{\max _{d} s\left(\tilde{F}_{d}^{n o r m}\right)}>0
$$

where $\tilde{F}_{d}$ is the same as in section 4. 
Proof. By homotopy invariance we can suppose that $f$ is a cell map of a finite cell complex. By definition the Nielsen number $N\left(f^{n}\right)$ is the number of non-zero terms in $L_{\pi}\left(f^{n}\right)$ (see section 4). The norm $\left\|L_{H}\left(f^{n}\right)\right\|$ is the sum of the absolute values of the indices of all the $n$-orbit classes $O^{n}$. It equals $\left\|L_{\pi}\left(f^{n}\right)\right\|$, the sum of the absolute values of the indices of all the fixed point classes of $f^{n}$, because any two fixed point classes of $f^{n}$ contained in the same $n$-orbit class $O^{n}$ must have the same index. From this we have $N\left(f^{n}\right) \leq\left\|L_{\pi}\left(f^{n}\right)\right\|=\left\|L_{H}\left(f^{n}\right)\right\|=\left\|\sum_{d}(-1)^{d}\left[\operatorname{Tr}\left(z \tilde{F}_{d}\right)^{n}\right]\right\| \leq \sum_{d}\left\|\left[\operatorname{Tr}\left(z \tilde{F}_{d}\right)^{n}\right]\right\| \leq$ $\sum_{d}\left\|\operatorname{Tr}\left(z \tilde{F}_{d}\right)^{n}\right\| \leq \sum_{d}\left\|\left(z \tilde{F}_{d}\right)^{n}\right\| \leq \sum_{d}\left\|\left(z \tilde{F}_{d}\right)\right\|^{n}$ (see [29]). The radius of convergence $R$ is given by the Cauchy-Hadamard formula:

$$
\frac{1}{R}=\limsup _{n}\left(\frac{N\left(f^{n}\right)}{n}\right)^{\frac{1}{n}}=\limsup _{n}\left(N\left(f^{n}\right)\right)^{\frac{1}{n}} .
$$

Therefore we have

$$
R=\frac{1}{\lim \sup _{n}\left(N\left(f^{n}\right)\right)^{\frac{1}{n}}} \geq \frac{1}{\max _{d}\left\|z \tilde{F}_{d}\right\|}>0
$$

The inequalities:

$$
\begin{aligned}
& N\left(f^{n}\right) \leq\left\|L_{\pi}\left(f^{n}\right)\right\|=\left\|L_{H}\left(f^{n}\right)\right\|=\left\|\sum_{d}(-1)^{d}\left[\operatorname{Tr}\left(z \tilde{F}_{d}\right)^{n}\right]\right\| \leq \sum_{d}\left\|\left[\operatorname{Tr}\left(z \tilde{F}_{d}\right)^{n}\right]\right\| \\
& \quad \leq \sum_{d}\left\|\operatorname{Tr}\left(z \tilde{F}_{d}\right)^{n}\right\| \leq \sum_{d} \operatorname{Tr}\left(\left(z \tilde{F}_{d}\right)^{n}\right)^{n o r m} \leq \sum_{d} \operatorname{Tr}\left(\left(z \tilde{F}_{d}\right)^{n o r m}\right)^{n} \leq \sum_{d} \operatorname{Tr}\left(\left(\tilde{F}_{d}\right)^{n o r m}\right)^{n}
\end{aligned}
$$

and the definition of spectral radius give the estimate:

$$
R=\frac{1}{\limsup _{n}\left(N\left(f^{n}\right)\right)^{\frac{1}{n}}} \geq \frac{1}{\max _{d} s\left(\tilde{F}_{d}^{\text {norm }}\right)}>0 .
$$

EXAmple 3. Let $X$ be a surface with boundary, and $f: X \rightarrow X$ be a map. Fadell and Husseini [9] devised a method of computing the matrices of the lifted chain map for surface maps. Suppose $\left\{a_{1}, \ldots, a_{r}\right\}$ is a free basis for $\pi_{1}(X)$. Then $X$ has the homotopy type of a bouquet $B$ of $r$ circles which can be decomposed into one 0-cell and $r$ 1-cells corresponding to the $a_{i}$, and $f$ has the homotopy type of a cellular map $g: B \rightarrow B$. By homotopy invariance, we can replace $f$ with $g$ in computations. The homomorphism $\tilde{f}_{*}$ : $\pi_{1}(X) \rightarrow \pi_{1}(X)$ induced by $f$ and $g$ is determined by the images $b_{i}=\tilde{f}_{*}\left(a_{i}\right), i=1, \ldots, r$. The fundamental group $\pi_{1}\left(T_{f}\right)$ has a presentation $\pi_{1}\left(T_{f}\right)=\left\langle a_{1}, \ldots, a_{r}, z\right| a_{i} z=z b_{i}, i=$ $1, \ldots, r\rangle$. Let

$$
D=\left(\frac{\partial b_{i}}{\partial a_{j}}\right)
$$

be the Jacobian in Fox calculus (see [4]). Then, as pointed out in [9], the matrices of the lifted chain map $\tilde{g}$ are

$$
\tilde{F}_{0}=(1), \quad \tilde{F}_{1}=D=\left(\frac{\partial b_{i}}{\partial a_{j}}\right) .
$$

Now, we can find estimates for the radius $R$ from (34) and (35).

REMARK 6. Let $X$ be a compact connected surface and $f: X \rightarrow X$ be a homeomorphism. Let $\chi(X)<0$. By Thurston's classification theorem [47] $f$ is isotopic to a homeomorphism $\phi$ such that either (1) $\phi$ is a periodic map; or (2) $\phi$ is a pseudo-Anosov 
map with stretching factor $\lambda>1$ (see section 3.3.1); or (3) $\phi$ is a reducible map, i.e. there is a system of disjoint simple closed curves in $\operatorname{int} X$ which is invariant under $\phi$ and which has a $\phi$-invariant tubular neighborhood $U$ such that each component of $X-U$ has negative Euler characteristic and on each $\phi$-component of $X-U, \phi$ satisfies 1 or 2 . Then, as follows from [19], $R=\frac{1}{\lambda}$, where $\lambda>1$ is the largest stretching factor of the pseudo-Anosov pieces (if there is no pseudo-Anosov piece then $\lambda=1$ ).

\section{Congruences for Reidemeister and Nielsen numbers}

8.1. Endomorphisms of the direct sum of a free Abelian and a finite group. In this section let $F$ be a finite group and $r$ a natural number. The group $G$ will be the direct sum

$$
G=\mathbb{Z}^{r} \oplus F .
$$

We shall describe the Reidemeister numbers of an endomorphism $\phi: G \rightarrow G$. The torsion elements of $G$ are exactly the elements of the finite, normal subgroup $F$. For this reason we have $\phi(F) \subset F$. Let $\phi^{\text {finite }}: F \rightarrow F$ be the restriction of $\phi$ to $F$, and let $\phi^{\infty}: G / F \rightarrow G / F$ be the induced map on the quotient group. We have proved in proposition 2 that

$$
R(\phi)=R\left(\phi^{\text {finite }}\right) \times R\left(\phi^{\infty}\right)
$$

We shall prove the following result:

Proposition 3. In the notation described above

$$
\# \operatorname{Fix}(\hat{\phi})=\# \operatorname{Fix}\left(\hat{\phi}^{\text {finite }}\right) \times \# \operatorname{Fix}\left(\hat{\phi}^{\infty}\right) \text {. }
$$

Proof. Consider the dual $\hat{G}$. This is the cartesian product of the duals of $\mathbb{Z}^{r}$ and $F$ :

$$
\hat{G}=\hat{\mathbb{Z}}^{r} \times \hat{F}, \quad \rho=\rho_{1} \otimes \rho_{2}
$$

where $\rho_{1}$ is an irreducible representation of $\mathbb{Z}^{r}$ and $\rho_{2}$ is an irreducible representation of $F$. Since $\mathbb{Z}^{r}$ is Abelian, all of its irreducible representations are 1-dimensional, so $\rho(v)$ for $v \in \mathbb{Z}^{r}$ is always a scalar matrix, and $\rho_{2}$ is the restriction of $\rho$ to $F$. If $\rho=\rho_{1} \otimes \rho_{2} \in \mathcal{S}(\phi)$ (see definition 1) then there is a matrix $T$ such that

$$
\rho \circ \phi=T \cdot \rho \cdot T^{-1} \text {. }
$$

This implies

$$
\rho^{\text {finite }} \circ \phi^{\text {finite }}=T \cdot \rho^{\text {finite }} \cdot T^{-1},
$$

so $\rho_{2}=\rho^{\text {finite }}$ is in $\mathcal{S}\left(\phi^{\text {finite }}\right)$. For any fixed $\rho_{2} \in \mathcal{S}\left(\phi^{\text {finite }}\right)$, the set of $\rho_{1}$ with $\rho_{1} \otimes \rho_{2} \in$ $\mathcal{S}\left(\phi^{\text {finite }}\right)$ is the set of $\rho_{1}$ satisfying

$$
\rho_{1}(M \cdot v) \rho_{2}(\psi(v))=T \cdot \rho_{1}(v) \cdot T^{-1}
$$

for some matrix $T$ independent of $v \in \mathbb{Z}^{r}$. Since $\rho_{1}(v)$ is a scalar matrix, the equation is equivalent to

$$
\rho_{1}(M \cdot v) \rho_{2}(\psi(v))=\rho_{1}(v),
$$

i.e.

$$
\rho_{1}((1-M) v)=\rho_{2}(\psi(v)) .
$$


Note that $\hat{\mathbb{Z}}^{r}$ is isomorphic to the torus $T^{n}$, and the transformation $\rho_{1} \rightarrow \rho_{1} \circ(1-M)$ is given by the action of the matrix $1-M$ on the torus $T^{n}$. Therefore the number of $\rho_{1}$ satisfying the last equation is the degree of the map $(1-M)$ on the torus, i.e. $|\operatorname{det}(1-M)|$. From this it follows that

$$
\# \operatorname{Fix}(\hat{\phi})=\# \operatorname{Fix}\left(\hat{\phi}^{\text {finite }}\right) \times|\operatorname{det}(1-M)| \text {. }
$$

As in the proof of proposition 3 we have $R\left(\phi^{\infty}\right)=|\operatorname{det}(1-M)|$. Since $\phi^{\infty}$ is an endomorphism of an Abelian group we have \# $\operatorname{Fix}\left(\hat{\phi}^{\infty}\right)=R\left(\phi^{\infty}\right)$. Therefore

$$
\# \operatorname{Fix}(\hat{\phi})=\# \operatorname{Fix}\left(\hat{\phi}^{\text {finite }}\right) \times \# \operatorname{Fix}\left(\hat{\phi}^{\infty}\right) \text {. }
$$

As a consequence we have the following

THEOREM 26. If $\phi$ be any endomorphism of $G$ where $G$ is the direct sum of a finite group $F$ and a finitely generated free Abelian group, then $R(\phi)=\# \operatorname{Fix}(\hat{\phi})$.

ProOF. Since $\phi^{\text {finite }}$ is an endomorphism of a finite group, by theorem 4 we have $R\left(\phi^{\text {finite }}\right)=\# \operatorname{Fix}\left(\hat{\phi}^{\text {finite }}\right)$. Since $\phi^{\infty}$ is an endomorphism of a finitely generated free Abelian group we have $R\left(\phi^{\infty}\right)=\# \operatorname{Fix}\left(\hat{\phi}^{\infty}\right)$ (see formula $(5)$ ). It now follows from propositions 2 and 3 that $R(\phi)=\# \operatorname{Fix}(\hat{\phi})$.

8.2. Endomorphisms of almost Abelian groups. In this section let $G$ be an almost Abelian and finitely generated group. A group will be called almost Abelian if it has an Abelian subgroup of finite index. We shall prove an analog of a theorem for almost Abelian groups found by the second author. It seems plausible that one could prove the same theorem for the so-called "tame" topological groups (see [32]). However we shall be interested mainly in discrete groups, and it is known that the discrete tame groups are almost Abelian.

We shall introduce the profinite completion $\bar{G}$ of $G$ and the corresponding endomorphism $\bar{\phi}: \bar{G} \rightarrow \bar{G}$. This is a compact totally disconnected group in which $G$ is densely embedded. The proof will then follow in three steps:

$$
R(\phi)=R(\bar{\phi}), \quad \# \operatorname{Fix}(\hat{\phi})=\# \operatorname{Fix}(\hat{\bar{\phi}}), \quad R(\bar{\phi})=\# \operatorname{Fix}(\hat{\bar{\phi}}) .
$$

If one omits the requirement that $G$ is almost Abelian then one can still show that $R(\phi) \geq R(\bar{\phi})$ and $\# \operatorname{Fix}(\hat{\phi}) \geq \# \operatorname{Fix}(\hat{\bar{\phi}})$. The third identity is a general fact for compact groups.

8.2.1. Compact groups. Here we shall prove the third of the above identities.

Let $K$ be a compact topological group and $\phi$ a continuous endomorphism of $K$. We define the number \# Fix ${ }^{\text {top }}(\hat{\phi})$ to be the number of fixed points of $\hat{\phi}$ in the unitary dual of $K$, where we only consider continuous representations of $K$. The number $R(\phi)$ is defined as usual.

TheOREM 27. For a continuous endomorphism $\phi$ of a compact group $K$ one has $R(\phi)=\# \operatorname{Fix}^{\text {top }}(\hat{\phi})$.

The proof uses the Peter-Weyl Theorem: 
TheOREm 28 (Peter-Weyl). If $K$ is compact then there is the following decomposition of the space $L^{2}(K)$ as a $K \oplus K$-module.

$$
L^{2}(K) \cong \bigoplus_{\lambda \in \hat{K}} \operatorname{Hom}_{C}\left(V_{\lambda}, V_{\lambda}\right)
$$

We also use Schur's Lemma:

LEMMA 10 (Schur). If $V$ and $W$ are two irreducible unitary representations then

$$
\operatorname{Hom}_{C K}(V, W) \cong\left\{\begin{array}{l}
0, V \nsubseteq W, \\
C, V \cong W .
\end{array}\right.
$$

Proof of Theorem 27. The $\phi$-conjugacy classes, being orbits of a compact group, are compact. Since there are only finitely many of them, they are also open subsets of $K$ and thus have positive Haar measure.

We embed $K$ in $K \oplus K$ by the map $g \mapsto(g, \phi(g))$. This makes $L^{2}(K)$ a $K$-module with a twisted action. By the Peter-Weyl Theorem we have (as $K$-modules)

$$
L^{2}(K) \cong \bigoplus_{\lambda \in \hat{K}} \operatorname{Hom}_{C}\left(V_{\lambda}, V_{\hat{\phi}(\lambda)}\right)
$$

We therefore have a corresponding decomposition of the space of $K$-invariant elements:

$$
L^{2}(K)^{K} \cong \bigoplus_{\lambda \in \hat{K}} \operatorname{Hom}_{C K}\left(V_{\lambda}, V_{\hat{\phi}(\lambda)}\right)
$$

We have used the well known identity $\operatorname{Hom}_{C}(V, W)^{K}=\operatorname{Hom}_{C K}(V, W)$. The left hand side consists of functions $f: K \rightarrow C$ satisfying $f\left(g x \phi(g)^{-1}\right)=f(x)$ for all $x, g \in K$. These are just functions on the $\phi$-conjugacy classes. The dimension of the left hand side is thus $R(\phi)$. On the other hand by Schur's Lemma the dimension of the right hand side is $\# \operatorname{Fix}^{\text {top }}(\hat{\phi})$.

8.2.2. Almost Abelian groups. Let $G$ be an almost Abelian group with an Abelian subgroup $A$ of finite index $[G: A]$. Let $A^{0}$ be the intersection of all subgroups of $G$ of index $[G: A]$. Then $A^{0}$ is an Abelian normal subgroup of finite index in $G$ and one has $\phi\left(A^{0}\right) \subset A^{0}$ for every endomorphism $\phi$ of $G$.

LEMMA 11. If $R(\phi)$ is finite then so is $R\left(\left.\phi\right|_{A^{0}}\right)$.

Proof. A $\phi$-conjugacy class is an orbit of the group $G$. A $\left.\phi\right|_{A^{0} \text {-conjugacy class is an }}$ orbit of the group $A^{0}$. Since $A^{0}$ has finite index in $G$ it follows that every $\phi$-conjugacy class in $A^{0}$ can be the union of at most finitely many $\left.\phi\right|_{A^{0} \text {-conjugacy classes. This proves }}$ the lemma.

Let $\bar{G}$ be the profinite completion of $G$ with respect to its normal subgroups of finite index. There is a canonical injection $G \rightarrow \bar{G}$ and the map $\phi$ can be extended to a continuous endomorphism $\bar{\phi}$ of $\bar{G}$. We shall write $\mathcal{R}(\phi)$ for the set of $\phi$-conjugacy classes of elements of $G$.

There is therefore a canonical map

$$
\mathcal{R}(\phi) \rightarrow \mathcal{R}(\bar{\phi})
$$


Since $G$ is dense in $\bar{G}$, the image of a $\phi$-conjugacy class $\{x\}_{\phi}$ is its closure in $\bar{G}$. From this it follows that the above map is surjective. We shall actually see that the map is bijective. This will then give us

$$
R(\phi)=R(\bar{\phi}) .
$$

However $\bar{\phi}$ is an endomorphism of the compact group $\bar{G}$ so by Theorem 27

$$
R(\bar{\phi})=\# \operatorname{Fix}^{\mathrm{top}}(\hat{\bar{\phi}}) .
$$

It thus suffices to prove the following two lemmas:

Lemma 12. If $R(\phi)$ is finite then \# $\operatorname{Fix}^{\text {top }}(\hat{\bar{\phi}})=\# \operatorname{Fix}(\hat{\phi})$.

Lemma 13. If $R(\phi)$ is finite then the map $\mathcal{R}(\phi) \rightarrow \mathcal{R}(\bar{\phi})$ is injective.

Proof of Lemma 12. By Mackey's Theorem (see [32]), every representation $\rho$ of $G$ is contained in a representation which is induced by a 1-dimensional representation $\chi$ of $A$. If $\rho$ is fixed by $\hat{\phi}$ then for all $a \in A^{0}$ we have $\chi(a)=\chi(\phi(a))$. Let $A^{1}=\left\{a \cdot \phi(a)^{-1}: a \in A^{0}\right\}$. By lemma $11 R\left(\left.\phi\right|_{A^{0}}\right)$ is finite and by lemma $1 R\left(\left.\phi\right|_{A^{0}}\right)=\left[A^{0}: A^{1}\right]$. Therefore $A^{1}$ has finite index in $G$. However we have shown that $\chi$ and therefore also $\rho$ is constant on cosets of $A^{1}$. Therefore $\rho$ has finite image, which implies that $\rho$ is the restriction to $G$ of a unique continuous irreducible representation $\bar{\rho}$ of $\bar{G}$. One verifies by continuity that $\hat{\bar{\phi}}(\bar{\rho})=\bar{\rho}$.

Conversely if $\bar{\rho} \in \mathcal{S}(\bar{\phi})$ then the restriction of $\bar{\rho}$ to $G$ is in $\mathcal{S}(\phi)$.

Proof of Lemma 13. We must show that the intersection with $G$ of the closure of $\{x\}_{\phi}$ in $\bar{G}$ is equal to $\{x\}_{\phi}$. We do this by constructing a coset of a normal subgroup of finite index in $G$ which is contained in $\{x\}_{\phi}$. For every $a \in A^{0}$ we have $x \sim_{\phi} x a$ if there is a $b \in A^{0}$ with $x^{-1} b x \phi(b)^{-1}=a$. It follows that $\{x\}_{\phi}$ contains a coset of the group $A_{x}^{2}:=\left\{x^{-1} b x \phi(b)^{-1}: b \in A^{0}\right\}$. It remains to show that $A_{x}^{2}$ has finite index in $G$.

Let $\psi(g)=x \phi(g) x^{-1}$. Then by corollary 1 we have $R(\psi)=R(\phi)$. This implies $R(\psi)<\infty$ and therefore by lemma 11 that $R\left(\left.\psi\right|_{A_{0}}\right)<\infty$. However by lemma 1 we have $R\left(\left.\psi\right|_{A_{0}}\right)=\left[A^{0}: A_{x}^{2}\right]$. This finishes the proof.

THEOREM 29. If $\phi$ is any endomorphism of $G$ where $G$ is an almost Abelian group, then $R(\phi)=\# \operatorname{Fix}(\hat{\phi})$.

Proof. This follows from lemmas 12, 13 and theorem 27.

8.3. Endomorphisms of nilpotent groups. In this section, we shall extend the computation of the Reidemeister number to endomorphisms of finitely generated torsion free nilpotent groups via topological techniques. Let $\Gamma$ be a finitely generated torsion free nilpotent group. It is well known [33] that $\Gamma=\pi_{1}(M)$ for some compact nilmanifold $M$. In fact, the rank (or Hirsch number) of $\Gamma$ is equal to $\operatorname{dim} M$, the dimension of $M$. Since $M$ is a $K(\Gamma, 1)$, every endomorphism $\phi: \Gamma \rightarrow \Gamma$ can be realized by a self-map $f: M \rightarrow M$ such that $f_{\#}=\phi$ and thus $R(f)=R(\phi)$.

THEOREM 30 ([18]). Let $\Gamma$ be a finitely generated torsion free nilpotent group of rank $n$. For any endomorphism $\phi: \Gamma \rightarrow \Gamma$ such that $R(\phi)$ is finite, there exists an endomorphism $\psi: \mathbb{Z}^{n} \rightarrow \mathbb{Z}^{n}$ such that $R(\phi)=\#$ Fix $\hat{\psi}$. 
8.4. Main theorem. The following lemma is useful for calculating Reidemeister numbers. It will also be used in the proof of the Main Theorem.

LEMMA 14. Let $\phi: G \rightarrow G$ be any endomorphism of any group $G$, and let $H$ be a subgroup of $G$ with the properties

$$
\phi(H) \subset H, \quad \forall x \in G \exists n \in \mathbb{N} \text { such that } \phi^{n}(x) \in H .
$$

Then $R(\phi)=R\left(\phi_{H}\right)$, where $\phi_{H}: H \rightarrow H$ is the restriction of $\phi$ to $H$.

Proof. Let $x \in G$. Then there is an $n$ such that $\phi^{n}(x) \in H$. From Lemma 1 it is known that $x$ is $\phi$-conjugate to $\phi^{n}(x)$. This means that the $\phi$-conjugacy class $\{x\}_{\phi}$ of $x$ has non-empty intersection with $H$.

Now suppose that $x, y \in H$ are $\phi$-conjugate, i.e. there is a $g \in G$ such that

$$
g x=y \phi(g) .
$$

We shall show that $x$ and $y$ are $\phi_{H}$-conjugate, i.e. we can find a $g \in H$ with the above property. First let $n$ be large enough that $\phi^{n}(g) \in H$. Then applying $\phi^{n}$ to the above equation we obtain

$$
\phi^{n}(g) \phi^{n}(x)=\phi^{n}(y) \phi^{n+1}(g) .
$$

This shows that $\phi^{n}(x)$ and $\phi^{n}(y)$ are $\phi_{H^{-}}$-conjugate. On the other hand, one knows by

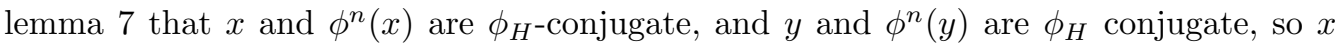
and $y$ must be $\phi_{H}$-conjugate.

We have shown that the intersection with $H$ of a $\phi$-conjugacy class in $G$ is a $\phi_{H^{-}}$ conjugacy class in $H$. We therefore have a map

$$
\text { Rest : } \mathcal{R}(\phi) \rightarrow \mathcal{R}\left(\phi_{H}\right), \quad\{x\}_{\phi} \mapsto\{x\}_{\phi} \cap H,
$$

This clearly has the two-sided inverse $\{x\}_{\phi_{H}} \mapsto\{x\}_{\phi}$. Therefore Rest is a bijection and $R(\phi)=R\left(\phi_{H}\right)$.

Corollary 12. Let $H=\phi^{n}(G)$. Then $R(\phi)=R\left(\phi_{H}\right)$.

Let $\mu(d), d \in \mathbb{N}$ be the Möbius function, i.e.

$$
\mu(d)= \begin{cases}1 & \text { if } d=1, \\ (-1)^{k} & \text { if } d \text { is a product of } k \text { distinct primes, } \\ 0 & \text { if } d \text { is not square-free. }\end{cases}
$$

Theorem 31 (Congruences for the Reidemeister numbers). Let $\phi: G \rightarrow G$ be an endomorphism of the group $G$ such that all numbers $R\left(\phi^{n}\right)$ are finite and let $H$ be a subgroup of $G$ with the properties

$$
\phi(H) \subset H, \quad \forall x \in G \exists n \in \mathbb{N} \text { such that } \phi^{n}(x) \in H .
$$

Suppose one of the following conditions is satisfied:

(I) $H$ is finitely generated Abelian,

(II) $H$ is finite,

(III) $H$ is a direct sum of a finite group and a finitely generated free Abelian group, or more generally

(IV) $H$ is finitely generated almost Abelian, or 
(V) $H$ is finitely generated, nilpotent and torsion free.

Then one has for all natural numbers $n$,

$$
\sum_{d \mid n} \mu(d) \cdot R\left(\phi^{n / d}\right) \equiv 0 \bmod n .
$$

ProOF. It follows immediately that, in cases I - IV, for every $n$

$$
R\left(\phi^{n}\right)=\# \operatorname{Fix}\left[{\hat{\phi_{H}}}^{n}: \hat{H} \rightarrow \hat{H}\right] .
$$

Let $P_{n}$ denote the number of periodic points of $\hat{\phi_{H}}$ of least period $n$. One sees immediately that

$$
R\left(\phi^{n}\right)=\# \operatorname{Fix}\left[{\hat{\phi_{H}}}^{n}\right]=\sum_{d \mid n} P_{d} .
$$

Applying Möbius' inversion formula, we have

$$
P_{n}=\sum_{d \mid n} \mu(d) R\left(\phi^{n / d}\right)
$$

On the other hand, we know that $P_{n}$ is always divisible by $n$, because $P_{n}$ is exactly $n$ times the number of $\hat{\phi_{H}}$-orbits in $\hat{H}$ of length $n$. In the case $\mathrm{V}$ when $H$ is finitely generated, nilpotent and torsion free, we know from theorem 30 that there exists an endomorphism $\psi: \mathbb{Z}^{n} \rightarrow \mathbb{Z}^{n}$ such that $R\left(\phi^{n}\right)=\#$ Fix $\hat{\psi}^{n}$. The proof then follows as in the previous cases.

REMARK 7. For finite groups, congruences for Reidemeister numbers follow from those of Dold for Lefschetz numbers since we have identified in remark 2 the Reidemeister numbers with the Lefschetz numbers of induced dual maps.

8.5. Congruences for Reidemeister numbers of a continuous map. Using corollary 1 we may apply the main theorem to the Reidemeister numbers of continuous maps.

THEOREM 32. Let $f: X \rightarrow X$ be a self-map such that all numbers $R\left(f^{n}\right)$ are finite. Let $f_{*}: \pi_{1}(X) \rightarrow \pi_{1}(X)$ be an induced endomorphism of the group $\pi_{1}(X)$ and let $H$ be a subgroup of $\pi_{1}(X)$ with the properties

$$
f_{*}(H) \subset H, \quad \forall x \in \pi_{1}(X) \exists n \in \mathbb{N} \text { such that } f_{*}^{n}(x) \in H .
$$

Suppose one of the following conditions is satisfied:

(I) $H$ is finitely generated Abelian,

(II) $H$ is finite,

(III) $H$ is a direct sum of a finite group and a finitely generated free Abelian group or more generally,

(IV) $H$ is finitely generated almost Abelian, or

(V) $H$ is finitely generated, nilpotent and torsion free.

Then one has for all natural numbers $n$,

$$
\sum_{d \mid n} \mu(d) \cdot R\left(f^{n / d}\right) \equiv 0 \bmod n .
$$


8.6. Congruences for Nielsen numbers of a continuous map

THEOREM 33. Suppose that there is a natural number $m$ such that $\tilde{f}_{*}^{m}(\pi) \subset I\left(\tilde{f}^{m}\right)$. If for every d dividing a certain natural number $n$ we have $L\left(f^{n / d}\right) \neq 0$, then one has for that particular $n$,

$$
\sum_{d \mid n} \mu(d) N\left(f^{n / d}\right) \equiv 0 \bmod n
$$

Proof. From the results of Jiang [28] we have that $N\left(f^{n / d}\right)=R\left(f^{n / d}\right)$ for the same particular $n$ and $\tilde{f}_{*}$ is eventually commutative. The result now follows from theorem 32 .

Corollary 13. Suppose $I\left(\mathrm{id}_{\tilde{X}}\right)=\pi$ and for every d dividing a certain natural number $n$ we have $L\left(f^{n / d}\right) \neq 0$. Then theorem 33 applies.

Corollary 14. Suppose that $X$ is aspherical, $f$ is eventually commutative and for every $d$ dividing a certain natural number $n$ we have $L\left(f^{n / d}\right) \neq 0$. Then theorem 33 applies.

EXAMPLE 4 . Let $f: T^{n} \rightarrow T^{n}$ be a hyperbolic endomorphism. Then for every natural $n$,

$$
\sum_{d \mid n} \mu(d) N\left(f^{n / d}\right) \equiv 0 \bmod n
$$

EXAMPLE 5. Let $g: M \rightarrow M$ be an expanding map [45] of the orientable smooth compact manifold $M$. Then $M$ is aspherical and is a $K\left(\pi_{1}(M), 1\right)$, and $\pi_{1}(M)$ is torsion free [45]. According to Shub [45] any lifting $\tilde{g}$ of $g$ has exactly one fixed point. From this and the covering homotopy theorem it follows that the fixed points of $g$ are pairwise inequivalent. The same is true for all iterates $g^{n}$. Therefore $N\left(g^{n}\right)=\# \operatorname{Fix}\left(g^{n}\right)$ for all $n$. So the sequence of the Nielsen numbers $N\left(g^{n}\right)$ of an expanding map satisfies the congruences as above.

THEOREM 34. Let $X$ be a connected, compact polyhedron with finite fundamental group $\pi$. Suppose that the action of $\pi$ on the rational homology of the universal cover $\tilde{X}$ is trival, i.e. for every covering translation $\alpha \in \pi, \alpha_{*}=\mathrm{id}: H_{*}(\tilde{X}, \mathbb{Q}) \rightarrow H_{*}(\tilde{X}, \mathbb{Q})$. If for every $d$ dividing a certain natural number $n$ we have $L\left(f^{n / d}\right) \neq 0$, then one has for that particular $n$,

$$
\sum_{d \mid n} \mu(d) N\left(f^{n / d}\right) \equiv 0 \bmod n
$$

Proof. From the results of Jiang [28] we have that $N\left(f^{n / d}\right)=R\left(f^{n / d}\right)$ for the same particular $n$. The result now follows from theorem 32 .

Lemma 15. Let $X$ be a polyhedron with finite fundamental group $\pi$ and let $p: \tilde{X} \rightarrow X$ be its universal covering. Then the action of $\pi$ on the rational homology of $\tilde{X}$ is trivial iff $H_{*}(\tilde{X} ; \mathbb{Q}) \cong H_{*}(X ; \mathbb{Q})$.

COROLlary 15. Let $\tilde{X}$ be a compact 1-connected polyhedron which is a rational homology $n$-sphere, where $n$ is odd. Let $\pi$ be a finite group acting freely on $\tilde{X}$ and let $X=\tilde{X} / \pi$. Then theorem 34 applies. 
Proof. The projection $p: \tilde{X} \rightarrow X=\tilde{X} / \pi$ is a universal covering space of $X$. For every $\alpha \in \pi$, the degree of $\alpha: \tilde{X} \rightarrow \tilde{X}$ must be 1 , because $L(\alpha)=0$ ( $\alpha$ has no fixed points). Hence $\alpha_{*}=\mathrm{id}: H_{*}(\tilde{X} ; \mathbb{Q}) \rightarrow H_{*}(\tilde{X} ; \mathbb{Q})$.

COROLlary 16. If $X$ is a closed 3-manifold with finite $\pi$, then theorem 34 applies.

Proof. $\tilde{X}$ is an orientable, simply connected manifold, hence a homology 3 -sphere. We apply corollary 15 .

EXAmple 6 . Let $X=L\left(m, q_{1}, \ldots, q_{r}\right)$ be a generalized lens space and $f: X \rightarrow X$ a continuous map with $f_{1 *}(1)=k$ where $|k| \neq 1$. Then for every natural $n$

$$
\sum_{d \mid n} \mu(d) N\left(f^{n / d}\right) \equiv 0 \bmod n .
$$

Proof. By corollary 15 we see that theorem 34 applies for lens spaces. Since $\pi_{1}(X)=$ $\mathbb{Z} / m \mathbb{Z}$, the map $f$ is eventually commutative. A lens space has the structure of a $\mathrm{CW}$ complex with one cell $e_{i}$ in each dimension $0 \leq i \leq 2 n+1$. The boundary map is given by $\partial e_{2 k}=m \cdot e_{2 k-1}$ for even cells, and $\partial e_{2 k+1}=0$ for odd cells. From this we may calculate the Lefschetz numbers:

$$
L\left(f^{n}\right)=1-k^{(l+1) n} \neq 0 .
$$

This is true for any $n$ as long as $|k| \neq 1$. The result now follows from theorem 34 .

REMARK 8. It is known that in the previous example

$$
N\left(f^{n}\right)=R\left(f^{n}\right)=\# \operatorname{Coker}\left(1-f_{1 *}^{n}\right)=\operatorname{gcd}\left(1-k^{n}, m\right)
$$

for every $n$. So we obtain a pure arithmetical fact: the sequence $n \mapsto \operatorname{gcd}\left(1-k^{n}, m\right)$ satisfies the congruences above for every natural $n$ if $|k| \neq 1$.

8.7. Some conjectures for wider classes of groups. For the case of almost nilpotent groups (i.e. groups with polynomial growth, in view of Gromov's theorem [25]) we believe that the congruences for the Reidemeister numbers are also true. We intend to prove this conjecture by identifying the Reidemeister number on the nilpotent part of the group with the number of fixed points in the direct sum of the duals of the quotients of successive terms in the central series. We then hope to show that the Reidemeister number of the whole endomorphism is a sum of numbers of orbits of such fixed points under the action of the finite quotient group (i.e. the quotient of the whole group by the nilpotent part). The situation for groups with exponential growth is very different. There one can expect the Reidemeister number to be infinite as long as the endomorphism is injective.

\section{Connection with Reidemeister torsion}

\subsection{Preliminaries}

9.1.1. Reidemeister torsion. Like the Euler characteristic, the Reidemeister torsion is algebraically defined. Roughly speaking, the Euler characteristic is a graded version of the dimension, extending the dimension from a single vector space to a complex of vector spaces. In a similar way, the Reidemeister torsion is a graded version of the absolute value of the determinant of an isomorphism of vector spaces. Let $d^{i}: C^{i} \rightarrow C^{i+1}$ be a cochain complex $C^{*}$ of finite dimensional vector spaces over $\mathbb{C}$ with $C^{i}=0$ for $i<0$ and large 
$i$. If the cohomology $H^{i}=0$ for all $i$ we say that $C^{*}$ is acyclic. If one is given positive densities $\Delta_{i}$ on $C^{i}$ then the Reidemeister torsion $\tau\left(C^{*}, \Delta_{i}\right) \in(0, \infty)$ for acyclic $C^{*}$ is defined as follows:

Definition 7. Consider a chain contraction $\delta^{i}: C^{i} \rightarrow C^{i-1}$, i.e. a linear map such that $d \circ \delta+\delta \circ d=i d$. Then $d+\delta$ determines a map $(d+\delta)_{+}: C^{+}:=\oplus C^{2 i} \rightarrow C^{-}:=\oplus C^{2 i+1}$ and a map $(d+\delta)_{-}: C^{-} \rightarrow C^{+}$. Since the map $(d+\delta)^{2}=i d+\delta^{2}$ is unipotent, $(d+\delta)_{+}$ must be an isomorphism. One defines $\tau\left(C^{*}, \Delta_{i}\right):=\left|\operatorname{det}(d+\delta)_{+}\right|($see [24]).

Reidemeister torsion is defined in the following geometric setting. Suppose $K$ is a finite complex and $E$ is a flat, finite dimensional, complex vector bundle with base $K$. We recall that a flat vector bundle over $K$ is essentially the same thing as a representation of $\pi_{1}(K)$ when $K$ is connected. If $p \in K$ is a basepoint then one may move the fibre at $p$ in a locally constant way around a loop in $K$. This defines an action of $\pi_{1}(K)$ on the fibre $E_{p}$ of $E$ above $p$. We call this action the holonomy representation $\rho: \pi \rightarrow G L\left(E_{p}\right)$. Conversely, given a representation $\rho: \pi \rightarrow G L(V)$ of $\pi$ on a finite dimensional complex vector space $V$, one may define a bundle $E=E_{\rho}=(\tilde{K} \times V) / \pi$. Here $\tilde{K}$ is the universal cover of $K$, and $\pi$ acts on $\tilde{K}$ by covering transformations and on $V$ by $\rho$. The holonomy of $E_{\rho}$ is $\rho$, so the two constructions give an equivalence of flat bundles and representations of $\pi$.

If $K$ is not connected then it is simpler to work with flat bundles. One then defines the holonomy as a representation of the direct sum of $\pi_{1}$ of the components of $K$. In this way, the equivalence of flat bundles and representations is recovered.

Suppose now that one has on each fibre of $E$ a positive density which is locally constant on $K$. In terms of $\rho_{E}$ this assumption just means $\left|\operatorname{det} \rho_{E}\right|=1$. Let $V$ denote the fibre of $E$.

Then the cochain complex $C^{i}(K ; E)$ with coefficients in $E$ can be identified with the direct sum of copies of $V$ associated to each $i$-cell $\sigma$ of $K$. The identification is achieved by choosing a basepoint in each component of $K$ and a basepoint from each $i$-cell. By choosing a flat density on $E$ we obtain a preferred density $\Delta_{i}$ on $C^{i}(K, E)$. One defines the R-torsion of $(K, E)$ to be $\tau(K ; E)=\tau\left(C^{*}(K ; E), \Delta_{i}\right) \in(0, \infty)$.

The Reidemeister torsion of an acyclic bundle $E$ on $K$ has many nice properties. Suppose that $A$ and $B$ are subcomplexes of $K$. Then we have a multiplicative law:

$$
\tau(A \cup B ; E) \cdot \tau(A \cap B ; E)=\tau(A ; E) \cdot \tau(B ; E)
$$

that is interpreted as follows. If three of the bundles $E|A \cup B, E| A \cap B, E|A, E| B$ are acyclic then so is the fourth and the equation (36) holds.

Another property is the simple homotopy invariance of the Reidemeister torsion. Suppose $K^{\prime}$ is a subcomplex of $K$ obtained by an elementary collapse of an $n$-cell $\sigma$ in $K$. This means that $K=K^{\prime} \cup \sigma \cup \sigma^{\prime}$ where $\sigma^{\prime}$ is an $(n-1)$-cell of $K$ such that $\partial \sigma^{\prime}=\sigma^{\prime} \cap K^{\prime}$ and $\sigma^{\prime} \subset \partial \sigma$, i.e. $\sigma^{\prime}$ is a free face of $\sigma$. So one can push $\sigma^{\prime}$ through $\sigma$ into $K^{\prime}$ giving a homotopy equivalence. Then $H^{*}(K ; E)=H^{*}\left(K^{\prime} ; E\right)$ and

$$
\tau\left(K ; E, D_{i}\right)=\tau\left(K^{\prime} ; E, D_{i}\right) .
$$

By iterating a sequence of elementary collapses and their inverses, one obtains a homotopy equivalence of complexes that is called simple. Plainly one has, by iterating (37), that the 
Reidemeister torsion is a simple homotopy invariant. In particular $\tau$ is invariant under subdivision. This implies that for a smooth manifold, one can unambiguously define $\tau\left(K ; E, D_{i}\right)$ to be the torsion of any smooth triangulation of $K$.

In the case $K=S^{1}$ is a circle, let $A$ be the holonomy of a generator of the fundamental group $\pi_{1}\left(S^{1}\right)$. One has that $E$ is acyclic iff $I-A$ is invertible and then

$$
\tau\left(S^{1} ; E\right)=|\operatorname{det}(I-A)| .
$$

Note that the choice of the generator is irrelevant as $I-A^{-1}=\left(-A^{-1}\right)(I-A)$ and $\left|\operatorname{det}\left(-A^{-1}\right)\right|=1$.

These three properties of the Reidemeister torsion are the analogues of the properties of Euler characteristic (cardinality law, homotopy invariance and normalization at a point), but there are differences. Since a point has no acyclic representations $\left(H^{0} \neq 0\right)$ one cannot normalize $\tau$ at a point as we do for the Euler characteristic, and so one must use $S^{1}$ instead. The multiplicative cardinality law for the Reidemeister torsion can be made additive just by using $\log \tau$, so the difference here is inessential. More important for some purposes is that the Reidemeister torsion is not an invariant under a general homotopy equivalence: as mentioned earlier this is in fact why it was first invented.

It might be expected that the Reidemeister torsion counts something geometric (like the Euler characteristic). D. Fried showed that it counts the periodic orbits of a flow and the periodic points of a map. We will show that the Reidemeister torsion counts the periodic point classes of a map (fixed point classes of the iterations of the map).

Some further properties of $\tau$ describe its behaviour under bundles.

Let $p: X \rightarrow B$ be a simplicial bundle with fiber $F$ where $F, B, X$ are finite complexes and $p^{-1}$ sends subcomplexes of $B$ to subcomplexes of $X$ over the circle $S^{1}$. We assume here that $E$ is a flat, complex vector bundle over $B$. We form its pullback $p^{*} E$ over $X$. Note that the vector spaces $H^{i}\left(p^{-1}(b), \mathbb{C}\right)$ with $b \in B$ form a flat vector bundle over $B$, which we denote $H^{i} F$. The integral lattice in $H^{i}\left(p^{-1}(b), \mathbb{R}\right)$ determines a flat density by the condition that the covolume of the lattice is 1 . We suppose that the bundle $E \otimes H^{i} F$ is acyclic for all $i$. Under these conditions D. Fried [24] has shown that the bundle $p^{*} E$ is acyclic, and

$$
\tau\left(X ; p^{*} E\right)=\prod_{i} \tau\left(B ; E \otimes H^{i} F\right)^{(-1)^{i}} .
$$

The opposite extreme is when one has a bundle $E$ on $X$ for which the restriction $E \mid F$ is acyclic. Then, for $B$ connected,

$$
\tau(X ; E)=\tau(F ; E \mid F)^{\chi(B)} .
$$

Suppose in (40) that $F=S^{1}$, i.e. $X$ is a circle bundle. Then (40) can be regarded as saying that

$$
\log \tau(X ; E)=\chi(B) \cdot \log \tau(F ; E \mid F)
$$

is counting the circle fibres in $X$ in the way that $\chi$ counts points in $B$, with a weighting factor of $\log \tau(F ; E \mid F)$.

9.2. The Reidemeister zeta function and the Reidemeister torsion of the mapping torus of the dual map. Let $f: X \rightarrow X$ be a homeomorphism of a compact polyhedron 
$X$. Let $T_{f}:=(X \times I) /(x, 0) \sim(f(x), 1)$ be the mapping torus of $f$. We shall consider the bundle $p: T_{f} \rightarrow S^{1}$ over the circle $S^{1}$. We assume here that $E$ is a flat, complex vector bundle with finite dimensional fibre and base $S^{1}$. We form its pullback $p^{*} E$ over $T_{f}$. Note that the vector spaces $H^{i}\left(p^{-1}(b), c\right)$ with $b \in S^{1}$ form a flat vector bundle over $S^{1}$, which we denote $H^{i} F$. The integral lattice in $H^{i}\left(p^{-1}(b), \mathbb{R}\right)$ determines a flat density by the condition that the covolume of the lattice is 1 . We suppose that the bundle $E \otimes H^{i} F$ is acyclic for all $i$. Under these conditions D. Fried [24] has shown that the bundle $p^{*} E$ is acyclic, and we have

$$
\tau\left(T_{f} ; p^{*} E\right)=\prod_{i} \tau\left(S^{1} ; E \otimes H^{i} F\right)^{(-1)^{i}}
$$

Let $g$ be the preferred generator of the group $\pi_{1}\left(S^{1}\right)$ and let $A=\rho(g)$ where $\rho: \pi_{1}\left(S^{1}\right) \rightarrow$ $G L(V)$. Then the holonomy around $g$ of the bundle $E \otimes H^{i} F$ is $A \otimes f_{i}^{*}$.

Since $\tau(E)=|\operatorname{det}(I-A)|$ it follows from (39) that

$$
\tau\left(T_{f} ; p^{*} E\right)=\prod_{i}\left|\operatorname{det}\left(I-A \otimes f_{i}^{*}\right)\right|^{(-1)^{i}} .
$$

We now consider the special case in which $E$ is one-dimensional, so $A$ is just a complex scalar $\lambda$ of modulus one. Then in terms of the rational function $L_{f}(z)$ we have [24]:

$$
\tau\left(T_{f} ; p^{*} E\right)=\prod_{i}\left|\operatorname{det}\left(I-\lambda \cdot f_{i}^{*}\right)\right|^{(-1)^{i}}=\left|L_{f}(\lambda)\right|^{-1}
$$

TheOREm 35. Let $\phi: G \rightarrow G$ be an automorphism of $G$, where $G$ is the direct sum of a finite group and a finitely generated free Abelian group. Then

$$
\tau\left(T_{\hat{\phi}} ; p^{*} E\right)=\left|L_{\hat{\phi}}(\lambda)\right|^{-1}=\left|R_{\phi}(\sigma \lambda)\right|^{(-1)^{r+1}},
$$

where $\lambda$ is the holonomy of the one-dimensional flat complex bundle $E$ over $S^{1}, r$ and $\sigma$ are the constants described in theorem 5.

Proof. We know from theorem 26 that $R\left(\phi^{n}\right)$ is the number of fixed points of the map $\hat{\phi}^{n}$. In general it is only necessary to check that the number of fixed points of $\hat{\phi}^{n}$ is equal to the absolute value of its Lefschetz number. We assume without loss of generality that $n=1$. We are assuming that $R(\phi)$ is finite, so the fixed points of $\hat{\phi}$ form a discrete set. We therefore have

$$
L(\hat{\phi})=\sum_{x \in \operatorname{Fix} \hat{\phi}} \operatorname{Index}(\hat{\phi}, x) .
$$

Since $\phi$ is a group endomorphism, the trivial representation $x_{0} \in \hat{G}$ is always fixed. Let $x$ be any fixed point of $\hat{\phi}$. Since $\hat{G}$ is a union of tori $\hat{G}_{0}, \ldots, \hat{G}_{t}$ and $\hat{\phi}$ is a linear map, we can shift any two fixed points to one another without altering the map $\hat{\phi}$. This gives us for any fixed point $x$ the equality

$$
\operatorname{Index}(\hat{\phi}, x)=\operatorname{Index}\left(\hat{\phi}, x_{0}\right)
$$

and so all fixed points have the same index. It is now sufficient to show that $\operatorname{Index}\left(\hat{\phi}, x_{0}\right)=$ \pm 1 . This follows because the map $\hat{\phi}: \hat{G}_{0} \rightarrow \hat{G}_{0}$ on the torus lifts to a linear map of the universal cover, which is a euclidean space. The index is then the sign of the determinant of the identity map minus this lifted map. This determinant cannot be zero, because $1-\hat{\phi}$ 
must have finite kernel by our assumption that the Reidemeister number of $\phi$ is finite (if $\operatorname{det}(1-\hat{\phi})=0$ then the kernel of $1-\hat{\phi}$ is a positive dimensional subspace of $\hat{G}$, and therefore infinite).

Corollary 17. Let $f: X \rightarrow X$ be a homeomorphism of a compact polyhedron $X$. If $\pi_{1}(X)$ is the direct sum of a finite group and a free Abelian group, then

$$
\tau\left(T_{\widehat{\left(f_{1 *}\right)}} ; p^{*} E\right)=\left|L_{\widehat{\left(f_{1 *}\right)}}(\lambda)\right|^{-1}=\left|R_{f}(\sigma \lambda)\right|^{(-1)^{r+1}},
$$

where $r$ and $\sigma$ are the constants described in theorem 9.

\section{Concluding remarks}

10.1. Reidemeister and Nielsen numbers and zeta functions modulo a normal subgroup. In the theory of (ordinary) fixed point classes, we work on the universal covering space. The group of covering transformations plays a key role. It is not surprising that this theory can be generalized to work on all regular covering spaces. Let $K$ be a normal subgroup of the fundamental group $\pi_{1}(X)$. Consider the regular covering $p_{K}: \tilde{X} / K \rightarrow X$ corresponding to $K$. A map $\tilde{f}_{K}: \tilde{X} / K \rightarrow \tilde{X} / K$ is called a lifting of $f: X \rightarrow X$ if $p_{K} \circ \tilde{f}_{K}=f \circ p_{K}$. We know from the theory of covering spaces that such liftings exist if and only if $f_{*}(K) \subset K$. If $K$ is a fully invariant subgroup of $\pi_{1}(X)$ (in the sense that every endomorphism sends $K$ into $K$ ) such as, for example, the commutator subgroup of $\pi_{1}(X)$, then there is a lifting of any continuous map. We may define the mod $K$-Reidemeister and Nielsen numbers (see [28]) and zeta functions (see [13]) and develop a similar theory by simply replacing $\tilde{X}$ and $\pi_{1}(X)$ by $\tilde{X} / K$ and $\pi_{1}(X) / K$ in every definition, every theorem and every proof, since everything was done in terms of liftings and covering translations.

\subsection{Minimal dynamical zeta function}

10.2.1. Radius of convergence of the minimal zeta function. In the Nielsen theory for periodic points, it is well known that $N\left(f^{n}\right)$ is often poor as a lower bound for the number of fixed points of $f^{n}$. A good homotopy invariant lower bound $N F_{n}(f)$, called the Nielsen type number for $f^{n}$, is defined in [28]. Consider any finite set of periodic orbit classes $\left\{O^{k_{j}}\right\}$ of varied period $k_{j}$ such that every essential periodic $m$-orbit class, $m \mid n$, contains at least one class in the set. Then $N F_{n}(f)$ is the minimal sum $\sum_{j} k_{j}$ for all such finite sets. Halpern (see [28]) has proved that for all $n N F_{n}(f)=\min \left\{\# \operatorname{Fix}\left(g^{n}\right) \mid g\right.$ has the same homotopy type as $f\}$. Recently, Jiang [29] found that as far as asymptotic growth rate is concerned, these Nielsen type numbers are no better than the Nielsen numbers.

LEMMA 16 ([29]).

$$
\limsup _{n}\left(N\left(f^{n}\right)\right)^{\frac{1}{n}}=\limsup _{n}\left(N F_{n}(f)\right)^{\frac{1}{n}} .
$$

Let us consider the minimal dynamical zeta function

$$
M_{f}(z):=\exp \left(\sum_{n=1}^{\infty} \frac{N F_{n}(f)}{n} z^{n}\right) .
$$


In [14] it was proved that $M_{f}(z)$ has positive radius of convergence $R$. Below we propose another proof of this fact and give an exact algebraic lower estimate for $R$.

TheOREM 36. For any continuous map $f$ of any compact polyhedron $X$ into itself the minimal zeta function has positive radius of convergence $R$ which admits the estimates

$$
\begin{gathered}
R \geq \exp (-h)>0, \\
R \geq \frac{1}{\max _{d}\left\|z \tilde{F}_{d}\right\|}>0,
\end{gathered}
$$

and

$$
R \geq \frac{1}{\max _{d} s\left(\tilde{F}_{d}^{n o r m}\right)}>0
$$

where $\tilde{F}_{d}$ and $h$ are the same as in section 6.1 .

Proof. The theorem follows from the Cauchy-Hadamard formula, lemma 11 and theorems 24 and 25 .

\subsection{Open questions}

QUESTION 1. For which spaces and maps does equality hold in (34)-(35)?

Sometimes, the minimal zeta function coincides with the Nielsen zeta function, for example, for a hyperbolic endomorphism of a torus and for an expanding map of an orientable smooth manifold (see [14]). This motivates the following

QUESTION 2. For which spaces and maps is the minimal zeta function a rational function? When is it a meromorphic function? When does it have a functional equation? What zeros and poles does it have?

Question 3. For which spaces and maps do we have a trace formula for Nielsen type numbers? When do we have the Dold congruences for these numbers? When does equality hold in the inequalities (45)-(47)?

The trace formulae which we have obtained in this article appear to be very similar to formulae arising in thermodynamical formalism. The relation of the radius of convergence with the entropy (theorem 24), Markov partition in the pseudo-Anosov case (theorem 22) and the relation of the Reidemeister zeta function to the Artin-Mazur zeta function on the unitary dual space (theorem 26) also indicate a connection with this theory. It is another open question to understand this connection.

\section{References}

[1] D. Anosov, The Nielsen number of maps of nilmanifolds, Russian Math. Surveys 40 (1985), 149-150.

[2] M. Artin and B. Mazur, On periodic points, Ann. of Math. 81 (1965), 82-99.

[3] I. Babenko and S. Bogatyi, Private communication.

[4] J. S. Birman, Braids, links and mapping class groups, Ann. Math. Studies 82, Princeton Univ. Press, Princeton, 1974. 
[5] R. BOwEN and O. LANFORD, Zeta functions of restrictions of the shift transformation, Proc. Global Anal. 1968, 43-49.

[6] J. Cheeger, Analytic torsion and the heat equation, Ann. of Math. 109 (1979), 259-322.

[7] A. Dold, Fixed point indices of iterated maps, Invent. Math. 74 (1983), 419-435.

[8] C. Epstein, The spectral theory of geometrically periodic hyperbolic 3-manifolds, Mem. AMS, 335 (1985).

[9] E. FAdell and S. Husseini, The Nielsen number on surfaces, in: Topological methods in nonlinear functional analysis, Contemp. Math. 21, AMS, 1983, 59-98.

[10] A. Fathi and M. Shub, Some dynamics of pseudo-Anosov diffeomorphisms, Astérisque 66-67 (1979), 181-207.

[11] A. L. Fel'shtyn, New zeta function in dynamics, in: Tenth Internat. Conf. on Nonlinear Oscillations, Varna, Abstracts of Papers, Bulgar. Acad. Sci., 1984, 208.

[12] A. L. FEL'SHTYN, A new zeta function in Nielsen theory and the universal product formula for dynamic zeta functions, Funktsional. Anal. i Prilozhen. 21 (2) (1987), 90-91 (in Russian); English transl.: Functional Anal. Appl. 21 (1987), 168-170.

[13] A. L. Fel'shtyn, Zeta functions in Nielsen theory, Funktsional. Anal. i Prilozhen. 22 (1) (1988), 87-88 (in Russian); English transl.: Functional Anal. Appl. 22 (1988), 76-77.

[14] A. L. FeL'Shtyn, New zeta functions for dynamical systems and Nielsen fixed point theory, in: Lecture Notes in Math. 1346, Springer, 1988, 33-55.

[15] A. L. FEL'Shtyn, The Reidemeister zeta function and the computation of the Nielsen zeta function, Colloq. Math. 62 (1991), 153-166.

[16] A. L. Fe'shtyn and R. Hill, Dynamical zeta functions, Nielsen theory and Reidemeister torsion, in: Nielsen Theory and Dynamical Systems, Contemp. Math. 152, AMS, 1993, 43-69.

[17] A. L. Fel'Shtyn and R. HiLl, The Reidemeister zeta function with applications to Nielsen theory and a connection with Reidemeister torsion, K-theory 8 (1994), 367-393.

[18] A. L. Fel'shtyn, R. Hill and P. Wong, Reidemeister numbers of equivariant maps, Topology Appl. 67 (1995), 119-131.

[19] A. L. Fel'shtyn and V. B. Pilyugina, The Nielsen zeta function, Funktsional. Anal. i Prilozhen. 19 (4) (1985), 61-67 (in Russian); English transl.: Functional Anal. Appl. 19 (1985), 300-305.

[20] J. Franks, Homology and Dynamical Systems, CMBS Regional Conf. Ser. Math. 49 (1982)

[21] W. Franz, Über die Torsion einer Überdeckung, J. Reine Angew. Math. 173 (1935), $245-254$.

[22] D. Fried, Periodic points and twisted coefficients, in: Lect. Notes in Math. 1007 (1983), 261-293.

[23] D. Fried, Homological identities for closed orbits, Invent. Math. 71 (1983), 219-246.

[24] D. FrIED, Lefschetz formula for flows, in: The Lefschetz centennial conference, Contemp. Math. 58, AMS, 1987, 19-69.

[25] M. Gromov, Groups of polynomial growth and expanding maps, Publ. Math. 53 (1981), $53-78$.

[26] M. Gromov, Hyperbolic groups, in: Essays in Group Theory, Math. Sci. Res. Inst. Publ. 8, 1987, 75-265.

[27] P. R. HEAth, Product formulae for Nielsen numbers of fibre maps, Pacific J. Math. 117 (2) (1985), 267-289.

[28] B. JiAng, Lectures on Nielsen Fixed Point Theory, Contemp. Math. 14, AMS, 1983. 
[29] B. JIANG, Estimation of the number of periodic orbits, Preprint of Universität Heidelberg, Mathematisches Institut, Heft 65, Mai 1993.

[30] B. JiAng and S. WANG, Lefschetz numbers and Nielsen numbers for homeomorphisms on aspherical manifolds, in: Topology - Hawaii, World Sci., Singapore, 1992, 119-136.

[31] S. Lang, Algebra, Addison-Wesley, 1993.

[32] A. A. KiRILlov, Elements of the Theory of Representations, Springer Verlag, 1976.

[33] A. MAL'CEv, On a class of homogeneous spaces, Izv. Akad. Nauk SSSR Ser. Mat. 13 (1949), 9-32 (in Russian).

[34] A. Manning, Axiom A diffeomorphisms have rational zeta function, Bull. London Math. Soc. 3 (1971), 215-220.

[35] J. Milnor, Infinite cyclic covers, in: Proc. Conf. "Topology of Manifolds" in Michigan 1967, 115-133.

[36] J. Milnor, A duality theorem for the Reidemeister torsion, Ann. of Math. 76 (1962), $137-147$.

[37] W. MüLleR, Analytic torsion and R-torsion of Riemannian manifolds, Adv. in Math. 28 (1978), 233-305.

[38] B. Norton-Odenthal, Ph. D. Thesis, University of Wisconsin, Madison, 1991.

[39] W. Parry and M. Pollicott, Zeta functions and the periodic structure of hyperbolic dynamics, Astérisque 187-188 (1990).

[40] D. RAY and I. Singer, R-torsion and the Laplacian on Riemannian manifolds, Adv. in Math. 7 (1971), 145-210.

[41] K. Reidemeister, Automorphism von Homotopiekettenringen, Math. Ann. 112 (1936), 586-593.

[42] G. DE RhAm, Complexes à automorphismes et homéomorphie différentiable, Ann. Inst. Fourier 2 (1950), 51-67.

[43] W. Rudin, Fourier Analysis on Groups, Interscience, 1962.

[44] D. Ruelle, Zeta function for expanding maps and Anosov flows, Invent. Math. 34 (1976), 231-242.

[45] M. Shub, Endomorphisms of compact differentiable manifolds, Amer. J. Math. 91 (1969), $175-179$

[46] H. Steinlein, Ein Satz über den Leray-Schauderschen Abbildungsgrad, Math. Z. 126 (1972), 176-208.

[47] W. P. Thurston, On the geometry and dynamics of diffeomorphisms of surfaces, Bull. AMS 19 (1988), 417-431.

[48] F. Wecken, Fixpunktklassen II, Math. Ann. 118 (1942), 216-234.

[49] A. WEIL, Numbers of solutions of equations in finite fields, Bull. AMS 55 (1949), 497-508.

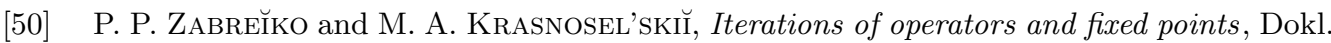
Akad. Nauk SSSR 196 (1971), 1006-1009 (in Russian). 